\title{
Periodic motion of Saturn's nightside plasma sheet
}

\author{
C. S. Arridge, ${ }^{1,2}$ N. André,,${ }^{3,4}$ K. K. Khurana, ${ }^{5}$ C. T. Russell, ${ }^{5}$ S. W. H. Cowley, ${ }^{6}$ \\ G. Provan, ${ }^{6}$ D. J. Andrews, ${ }^{6}$ C. M. Jackman, ${ }^{2,7,8}$ A. J. Coates, ${ }^{1,2}$ E. C. Sittler, ${ }^{9}$ \\ M. K. Dougherty, ${ }^{7}$ and D. T. Young ${ }^{10}$
}

Received 11 May 2011; revised 8 August 2011; accepted 11 August 2011; published 5 November 2011.

[1] Saturn's magnetosphere is replete with magnetospheric periodicities; magnetic fields, plasma parameters, energetic particle fluxes, and radio emissions have all been observed to vary at a period close to that of Saturn's assumed sidereal rotation rate. In particular, periodicities in Saturn's magnetotail can be interpreted in terms of periodic vertical motion of Saturn's outer magnetospheric plasma sheet. The phase relationships between periodicities in different measurable quantities are a key piece of information in validating the various published models that attempt to relate periodicities in different quantities at different locations. It is important to empirically extract these phase relationships from the data in order to distinguish between these models, and to provide further data on which to base new conceptual models. In this paper a simple structural model of the flapping of Saturn's plasma sheet is developed and fitted to plasma densities in the outer magnetosphere, measured by the Cassini electron spectrometer. This model is used to establish the phase relationships between magnetic field periodicities in the cam region of the magnetosphere and the flapping of the plasma sheet. We find that the plasma sheet flaps in phase with $\mathrm{B}_{\mathrm{r}}$ and $\mathrm{B}_{\theta}$ and in quadrature with the $\mathrm{B}_{\varphi}$ component in the core/cam region. The plasma sheet phase also has a strong local time asymmetry. These results support some conceptual periodicity models but are in apparent contradiction with others, suggesting that future work is required to either modify the models or study additional phase relationships that are important for these models.

Citation: Arridge, C. S., et al. (2011), Periodic motion of Saturn's nightside plasma sheet, J. Geophys. Res., 116, A11205, doi:10.1029/2011JA016827.

\section{Introduction}

[2] Despite the widely studied near axisymmetry of Saturn's internal magnetic field, periodicities have been observed in Saturn's magnetosphere since the epoch of the Pioneer 11 and Voyager flybys. Perhaps the most studied periodicities in Saturn's magnetosphere are those observed in kilometric radio emissions emitted from the auroral regions, known as Saturn Kilometric Radiation (SKR). Voyager measurements

\footnotetext{
${ }^{1}$ Mullard Space Science Laboratory, University College London, Dorking, UK.

${ }^{2}$ Centre for Planetary Sciences at UCL/Birkbeck, London, UK.

${ }^{3}$ Institut de Recherche en Astrophysique et Planétologie, CNRS, Toulouse, France.

${ }^{4}$ Institut de Recherche en Astrophysique et Planétologie, Université de Toulouse, UPS-OMP, Toulouse, France.

${ }^{5}$ Institute for Geophysics and Planetary Physics, University of California, Los Angeles, California, USA.

${ }^{6}$ Department of Physics and Astronomy, University of Leicester, Leicester, UK.

${ }^{7}$ Blackett Laboratory, Imperial College London, London, UK.

${ }^{8}$ Department of Physics and Astronomy, University College London, London, UK.

${ }^{9}$ NASA Goddard Space Flight Center, Greenbelt, Maryland, USA.

${ }^{10}$ Southwest Research Institute, San Antonio, Texas, USA.

Copyright 2011 by the American Geophysical Union. 0148-0227/11/2011JA016827
}

showed that SKR pulsed at a particular period that was rapidly adopted as the rotation rate of Saturn's deep interior, and a longitude system, known as the Saturn Longitude System (SLS), was constructed based on this rotation period [Desch and Kaiser, 1981]. Subsequently it has been shown that not only does the SKR period drift in time [Gurnett et al., 2005, and references therein] but also that emissions from the northern and southern auroral regions are modulated at different periods [Gurnett et al., 2009; Lamy, 2011]. During southern hemisphere summer the period of SKR emissions from the southern auroral zone were modulated at a longer period than those from the north. During the approach to equinox these two periods started to converge and after equinox in 2009 they became equal and then reversed, such that the emissions from the southern auroral zone were at a shorter period than those from the north [Gurnett et al., 2010; Lamy, 2011].

[3] The phase of the SKR periodicity can be used to organize fields and particles measurements made inside Saturn's magnetosphere. Two modified SLS systems known as SLS2 and SLS3 were constructed to reflect the observed drifting SKR period and are variable period systems [Kurth et al., 2008, and references therein]. The SLS2 system is valid from 1 January 2004 to 28 August 2006 and SLS3 covers a longer time period from 1 January 2004 to 10 August 2007. 
In their region of overlap the two variable period systems (SLS2 and SLS3) give equivalent results (to within the model uncertainty of $\sim 10^{\circ}$ ). The SLS3 system is defined as a westward longitude system such that longitude increases with time for an inertial observer. The signal from the southern auroral zone is stronger than that from the north and so before the discovery of separate northern and southern SKR periods the detected period was that of the southern zone. Therefore, SLS2 and SLS3 give the period of SKR emissions from the southern auroral zone. The difference between the separated southern phase from Lamy [2011] and SLS3 is small $\left( \pm \sim 20^{\circ}\right)$ over the period of interest in this paper [Andrews et al., 2010b].

[4] This phase model has been used to demonstrate that the southern SKR emissions have a distinct phase relationship with periodicities in Saturn's magnetosphere in the location of the auroral oval [Nichols et al., 2008; Provan et al., 2009b], magnetic fields [e.g., Espinosa et al., 2003a; Southwood and Kivelson, 2007; Andrews et al., 2008; Provan et al., 2009a], energetic particles [e.g., Carbary and Krimigis, 1982; Carbary et al., 2007b], plasma [e.g., Gurnett et al., 2007; Arridge et al., 2008a; Khurana et al., 2009; Burch et al., 2008, 2009], energetic neutral atoms (ENAs) [Carbary et al., 2008], and the location of the magnetopause [e.g., Clarke et al., 2010]. Since the discovery of distinct southern and northern SKR periods it has been shown that magnetic field perturbations on open field lines in the northern hemisphere are organized by the northern auroral zone SKR phase, and those in the southern hemisphere by the southern SKR phase [Andrews et al., $2010 \mathrm{~b}$. In addition, the near equatorial magnetic field oscillations in the inner and middle magnetosphere are also organized by the southern SKR phase [Andrews et al., 2010b] in the period leading up to equinox.

[5] Espinosa et al. [2003b] used the analogy of a mechanical camshaft to interpret how an anomaly located close to the planet could generate periodicities in the inner magnetosphere that also propagated outward to affect the whole magnetosphere. This anomaly was originally taken to be a high-degree internal magnetic field anomaly, but Cassini era interpretations are not so restrictive [e.g., Southwood and Kivelson, 2007]. Reflecting this analogy, the region inside of $\sim 15 \mathrm{R}_{\mathrm{S}}$ (where $1 \mathrm{R}_{\mathrm{S}}=60,268 \mathrm{~km}$ ) is referred to as the cam region and has been studied in detail using magnetic field and plasma data. Gurnett et al. [2007] used the SLS2 system to order total electron number density $\mathrm{n}_{\mathrm{e}}$ and azimuthal magnetic field $\mathrm{B}_{\varphi}$ (in a spherical polar coordinate system) between 3 and $5 \mathrm{R}_{\mathrm{S}}$ in the cam (out to $24 \mathrm{R}_{\mathrm{S}}$ for $\mathrm{B}_{\varphi}$ ). They showed that $\mathrm{n}_{\mathrm{e}}$ and $\mathrm{B}_{\varphi}$ were modulated with SLS2 longitude and both peaked at a longitude of $\sim 330^{\circ}$. Magnetic field data has also been studied by a number of authors showing that the radial and polar magnetic field components exhibit modulations that have a specific phase relationship with southern SKR. In particular, it is known that everywhere inside of $15 \mathrm{R}_{\mathrm{S}}$ the radial and polar magnetic field components are in phase with each other, but are in leading quadrature with $\mathrm{B}_{\varphi}$ [Southwood and Kivelson, 2007; Andrews et al., 2008, 2010b; Provan et al., 2009a].

[6] There is also an associated periodic perturbation in the $\mathrm{B}_{\theta}$ component of the field that has been related to the presence of a rotating partial ring current in the cam region [Khurana et al., 2009; Provan et al., 2009a; Brandt et al., 2010]. In terms of the perturbation field $\Delta B_{\theta}$ the ring cur- rent (symmetric+asymmetric) is strongest when the perturbation field is at a negative extrema, and is weakest when the perturbation field is the weakest corresponding to weaker azimuthal currents. Numerous studies have shown that the perturbations in $B_{\theta}$ are in phase with those in $B_{r}$ such that when $\Delta B_{r}>0$ (away from Saturn) the ring current is weak $\left(\Delta B_{\theta}\right.$ is small) and when $\Delta B_{r}<0$ (toward Saturn) the ring current is strong ( $\Delta \mathrm{B}_{\theta}$ is large and negative) [e.g., Andrews et al., 2008, 2010a, Provan et al., 2009a].

[7] Arridge et al. [2008a] studied electron densities (0.5 eV to $26 \mathrm{keV}$ ) in Saturn's equatorial plane near Titan's orbit. The electron density was found to be modulated with SLS3 longitude with density maxima that were in phase with those reported by Gurnett et al. [2007] and Carbary et al. [2007b]. The identified asymmetry was found to be consistent with periodic vertical motion of the plasma sheet. In the outer magnetosphere from near Titan's orbit to $\sim 40 \mathrm{R}_{\mathrm{S}}$, Carbary et al. [2007b] examined fluxes of energetic electrons and found a modulation that exhibited a longitudinal delay that increased with radial distance thus forming a retrograde spiral feature in the outer magnetosphere with a gradient between 2.7 and $4.7^{\circ} \mathrm{R}_{\mathrm{S}}^{-1}$. Interestingly, this spiral shape was fairly consistent until June 2006, when their results appeared to show a $\sim 180^{\circ}$ shift in the prime meridian of this spiral. Carbary et al. [2007b] discussed possible physical origins for this inferred shift but were careful to point out that this could be an artifact due to a change in viewing geometry on higher latitude orbits after June 2006. The production of spiral features, associated with Saturn's magnetospheric periodicities, by radial phase delay effects was first discussed by Espinosa et al. [2003b], and the presence of such effects were demonstrated in Cassini magnetometer data by Cowley et al. [2006]. The use of SLS3 in these studies shows that at low (magnetic) latitudes the modulations are in phase with the southern SKR period, similar to the equatorial cam field [Andrews et al., 2010b].

[8] Burch et al. [2009] have also studied plasma periodicities in the outer magnetosphere and identified a similar longitudinal delay to Carbary et al. [2007b] but instead interpreted these as the periodic passage of plasmoids, using observations of northward magnetic fields on the trailing edge of each pulse of plasma as evidence of the plasmoid. Jackman et al. [2009a] have emphasized the clear difference between the periodic plasma and magnetic field signatures in the magnetotail, and unambiguously detected plasmoids. They went on to show that the northward field signatures described by Burch et al. [2009] can naturally arise in a wavy (flapping) magnetodisc model, where both radial and azimuthal currents flow, due to the periodically changing orientation of the current sheet with respect to the coordinate system used to study the data. Jackman et al. [2009b] studied nine plasmoids observed in magnetic field data and found that their release time occurred during the rising phase of (southern period) SKR for eight out of the nine plasmoids. Thus it appears that reconnection and associated plasmoids may occur at a preferential longitude (phase of SKR), but the evidence based on magnetic field data suggests that they occur much less frequently than once per planetary rotation, and that the periodic field signatures reported are purely due to current sheet flapping.

[9] In support of a flapping paradigm, Carbary et al. [2008] have used ENA observations to show a periodic tilting of the 


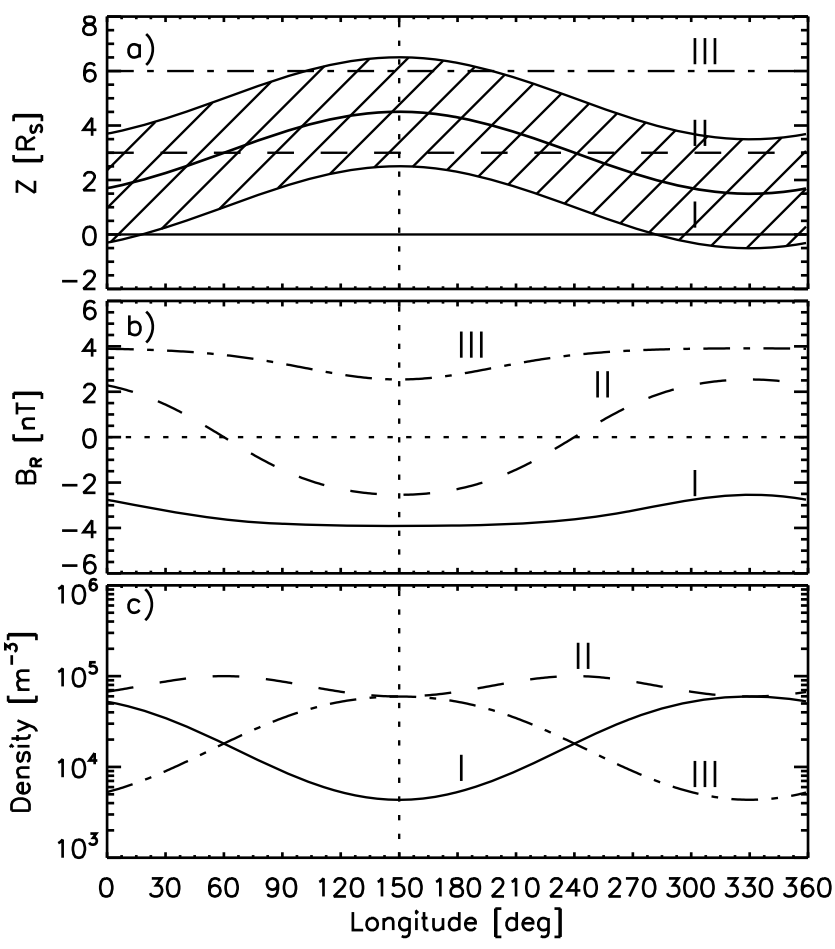

Figure 1. (a) Schematic illustration of three different trajectories (identified by Roman numerals) about a flapping plasma sheet, as a function of subspacecraft longitude. (b and c) The density and magnetic field profiles, estimated using a Harris neutral sheet model, show the profiles expected from each trajectory. If one uses the maxima in density or equivalently the maxima in particle flux, to establish the prime meridian for the oscillation, one will get very different longitudes depending on which trajectory is chosen.

plasma sheet, and Provan et al. [2009b] show how periodic tilting of the plasma sheet is linked in phase with the cam field and auroral oval oscillations. Several authors have noted that plasma sheet periodicities in the tail region can be understood in terms of a flapping plasma sheet and have developed conceptual models to attempt to understand how this can arise [e.g., Carbary et al., 2007a; Southwood and Kivelson, 2007; Khurana et al., 2009; Provan et al., 2009a]. These models all make particular predictions about how the motion of the tail should relate to the cam and SKR emissions.

[10] In studying the motion of the tail plasma sheet it is important to account for the geometry of the plasma sheet since it has been known for some time that the location of a spacecraft with respect to an oscillating plasma sheet can radically change the apparent behavior of the sheet [e.g., Vasyliūnas et al., 1997; Waldrop et al., 2005]. A spacecraft located slightly to the south of the plasma sheet mean location will see a completely different longitudinal behavior to a spacecraft located to the north of the plasma sheet mean location. Figure 1 illustrates this schematically with three imaginary spacecraft trajectories (indicated by Roman numerals) about an oscillating sheet and shows the observed magnetic field and density profiles in Figures $1 \mathrm{~b}$ and 1c. Clearly, the phase relationship derived from density data from trajectories I and III would be quite different and the actual trajectory taken by the spacecraft must be considered when inferring any phase relationship.

[11] In this paper no attempt is made to construct a physical model for the origin of the periodicities in the outer magnetosphere. Instead, the aim is to use simple structural models for the geometry and flapping of the plasma sheet to examine periodicities in the outer magnetosphere. Modeling of the geometry of the Jovian plasma sheet [e.g., Khurana and Schwarzl, 2005; Kivelson et al., 1978] uses the locations of the crossings to fit models of plasma sheet structure. Clear and sharp plasma sheet crossings are not particularly common in the Cassini data at Saturn, and so we use a global approach to make the most of the data. This simple model is used to establish the phase relationship between the motion of the plasma sheet, SKR, and magnetic and density periodicities in the "cam" or "core" region in the inner magnetosphere, to investigate the spiral patterns reported by Carbary et al. [2007b], to search for further evidence of an abrupt shift in the spiral beyond day 200 of 2006, and to search for evidence that supports or refutes the published physical models [Carbary et al., 2007a; Southwood and Kivelson, 2007; Khurana et al., 2009; Provan et al., 2009a].

[12] In this paper we analyze data from Cassini's passes through the magnetotail in 2006 where the spacecraft was close to the center of the oscillating plasma sheet. This provides a unique data set to establish phase relationships between the motion of the plasma sheet and other periodicities in the Saturnian magnetosphere. We use phase models derived from near equatorial magnetic data [Andrews et al., 2008; Provan et al., 2009a] and SLS3 and hence derive a relationship between the motion of the plasma sheet and oscillations related to the southern auroral zone. We demonstrate that (1) low (magnetic) and southern magnetic latitude plasma sheet oscillations are in phase with the southern SKR and near equatorial cam oscillations, (2) these oscillations are delayed in phase producing a retrograde spiral similar to the energetic particle observations, and (3) that the phase of the plasma sheet motions has a local time dependence similar to the core magnetic field.

[13] This paper is arranged into five sections; section 2 presents an overview of the data and evidence for vertical plasma sheet motion. Selected orbits are examined to exemplify certain features of the periodicity. In section 3 a simple structural model for the plasma sheet flapping is described, and the modeling methodology is discussed, with particular emphasis on how this model is fitted to the in situ Cassini data. Fits of the model to Cassini data are presented in section 5, where intervals that show good and poor agreements with the model are highlighted. The paper concludes with a discussion of our results and their implications in section 5 .

\section{Observations of Periodicities in Saturn's Outer Magnetosphere}

[14] Figure 2 presents an overview of periodicities in Saturn's magnetotail from Cassini's revolution 26 during July 2006. During this period Cassini was located close to the equator at a distance of $30-40 R_{S}$ in the post-midnight sector. The radial and azimuthal components of the field have an anti-phase relationship consistent with Cassini being 


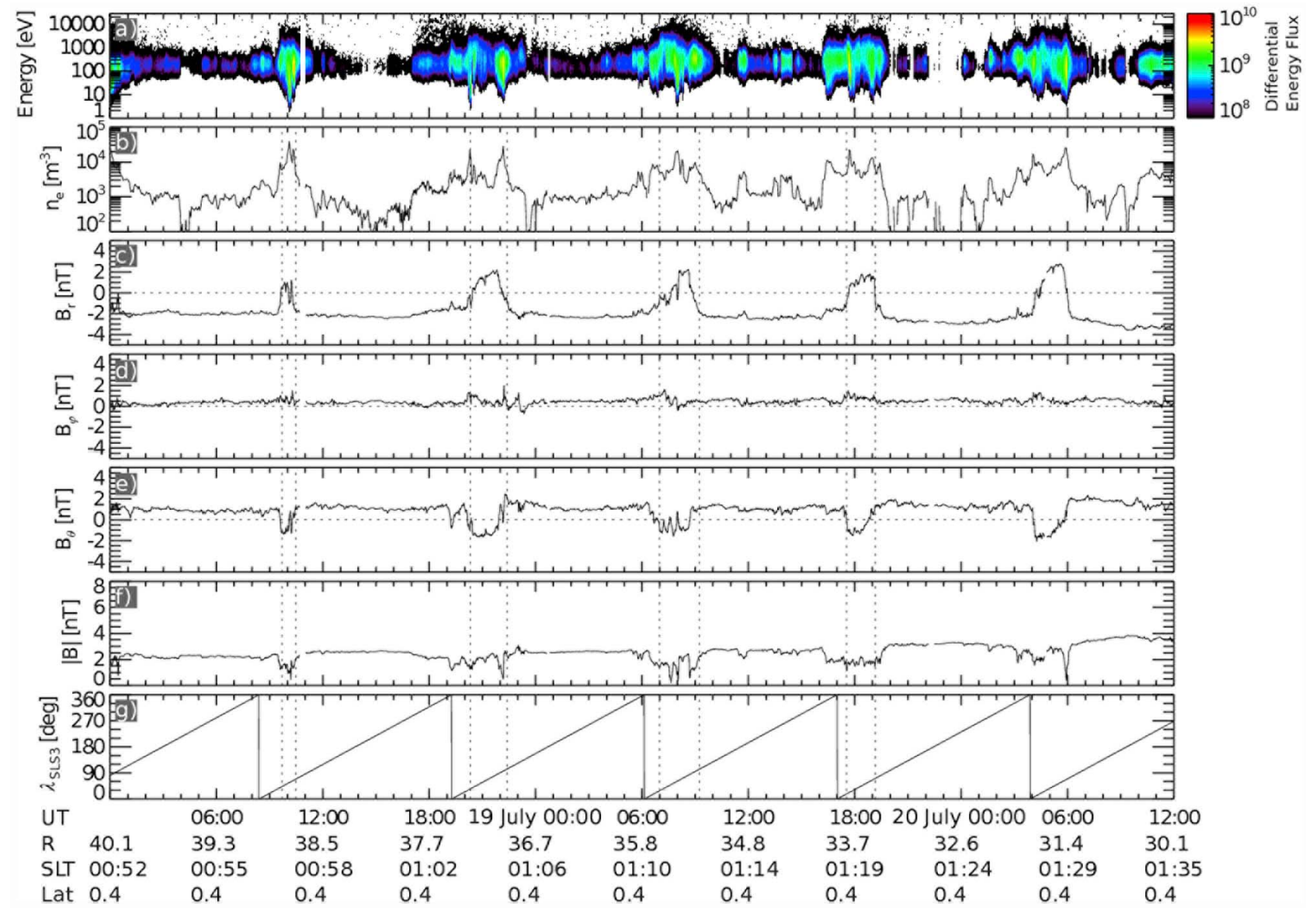

Figure 2. Overview of periodicities in Saturn's post-midnight current and plasma sheet from July 2006. (a) A time-energy electron spectrogram measured by CAPS/ELS in units of differential energy flux $\left(\mathrm{eV} \mathrm{m} \mathrm{m}^{-2} \mathrm{~s}^{-1} \mathrm{sr}^{-1} \mathrm{eV}^{-1}\right.$ ), which has been processed to remove trapped spacecraft photoelectrons, (b) electron number densities obtained by numerical integration of the measured spectra, $(\mathrm{c}-\mathrm{e})$ three components of the magnetic field in spherical polar (KRTP) coordinates, (f) field magnitude, and (g) SLS3 longitude. The vertical gray dashed lines identify crossings of the magnetic equator. In Figure $2 \mathrm{~g}$, the vertical dashed black lines denote an SLS3 longitude of $330^{\circ}$, matching the heavy sector identified by Gurnett et al. [2007]. The ephemeris information at the bottom shows the radial distance from Saturn (in $\mathrm{R}_{\mathrm{S}}$ ), the local time of the spacecraft (SLT) in hours and minutes, and the latitude of the spacecraft in degrees.

located on field lines connected to plasma which is in lagging corotation with Saturn's ionosphere. Current sheet crossings can be identified by a reversal in the magnetic field tangential to the current sheet $\mathbf{B}_{\mathbf{t}}$ [Vasyliūnas, 1983]. For moderately distorted current sheet geometries the $\mathrm{B}_{\mathrm{r}}$ and $\mathrm{B}_{\varphi}$ components of the field approximate the tangential field, and so changes in the signs of $\mathrm{B}_{\mathrm{r}}$ and $\mathrm{B}_{\varphi}$ can be used to identify current sheet crossings, and approaches to the current sheet can be identified by a weakening in $\left|\mathrm{B}_{\mathrm{r}}\right|$ and $\left|\mathrm{B}_{\varphi}\right|$. The polar component of the magnetic field is small and varies little as the spacecraft enters and leaves the current sheet, consistent with $\nabla \cdot \mathbf{B}=0$ near a thin current sheet. An exception to this is that flapping of the sheet can produce oscillations in the magnetic field component normal to the sheet due to changes in geometry, as discussed in section 1 [e.g., Jackman et al., 2009a]. These oscillations can be seen in the $\mathrm{B}_{\theta}$ component of the field as small increases in $\mathrm{B}_{\theta}$ at the leading edge and decreases and occasional reversals (northward turnings) in $\mathrm{B}_{\theta}$ on the trailing edge (compare with Figure 4 of Jackman et al. [2009a]).
[15] At each current sheet crossing (denoted by the vertical dashed gray lines in Figure 2), the electron number density and warm plasma electron flux maximize, consistent with a plasma sheet associated with the current sheet. A number of the crossings exhibit double-peak structures where the radial field changes sign and the spacecraft enters the northern magnetic hemisphere, before the spacecraft returns to the southern magnetic hemisphere. During these crossings the electron fluxes maximize near to $B_{r}=0$ and fall as $B_{r}$ rises to larger positive values, before maximizing once again as the spacecraft returns to the center of the plasma sheet and falls as the spacecraft moves back out into the low density outer plasma sheet and southern lobe. This "double-peak" behavior is also visible in the magnetic field strength, where the field strength exhibits a minimum at the plasma sheet center consistent with diamagnetic effects and a large plasma beta at the center of the sheet.

[16] Hence, the behavior of the magnetic field and particles is consistent with the passage through a thin current and 
plasma sheet. The SLS3 longitude in Figure $2 \mathrm{~g}$ shows that these crossings are encountered once per rotation. If Cassini were located at the center of the plasma sheet oscillation, two current sheet crossings would be observed per $360^{\circ}$ of SLS3 longitude: a north-to-south lobe crossing and another a south-to-north lobe crossing. These crossings would be separated in longitude by $180^{\circ}$. The approaches to the current sheet, and small longitudinal separation of the double-peaked current sheet crossings indicate that Cassini is located to the south of the mean sheet location. Some longitudinal shifts, of several tens of degrees in magnitude, are observed in the locations of these crossings and might be related to solar wind effects that can shift the mean location of the plasma sheet, or might be related to "jitter" in the underlying periodic mechanism [e.g., Andrews et al., 2008], possibly also related to the beating effects discussed by Provan et al. [2011].

[17] Since the Kronographic latitude of Cassini remains approximately constant near zero during the whole interval presented in Figure 2 and given the evidence that Cassini is periodically immersed in a thin current sheet, sometimes traversing the center plane of the sheet, one can conclude that Saturn's plasma sheet is undergoing vertical oscillations that have some phase relationship with southern SKR and cam region fields [e.g., Andrews et al., 2008; Arridge et al., 2008b] and particles [e.g., Gurnett et al., 2007].

\section{Simple Structural Model for Tail Periodicities}

[18] Arridge et al. [2008b] have shown that during Cassini's nominal mission (2004-2008), Saturn's magnetospheric current sheet was bent into a bowl shape due to its interaction with the solar wind. This deformation means that Cassini's equatorial orbits have typically been located below the mean location of the plasma sheet. Arridge et al. [2008b] presented a simple model for the mean location of the sheet using a local time symmetric expression that gave the height of the current sheet above Saturn's equatorial plane as a function of cylindrical radial distance, $\rho$, hinging distance, $\mathrm{r}_{\mathrm{H}}$, and the latitude of the Sun, $\theta_{\mathrm{SUN}}$.

$$
z_{C S}(\rho)=\left[\rho-r_{H} \tanh \left(\frac{\rho}{r_{H}}\right)\right] \tan \theta_{S U N} .
$$

To model the geometry of the observed flapping of the plasma sheet, this expression was modified to include a periodic term, which is a function of some phase $\Psi$, the purpose of which is to introduce a tilt, $\theta_{\mathrm{TILT}}$, to the sheet beyond a distance $\rho_{0}$. Before we discuss this periodic term, we first describe the phase models used to construct $\Psi$.

[19] Kurth et al. [2008, and references therein] used observations of a drifting SKR period to define a timedependent longitude system (e.g., SLS3) that was tied to the phase of SKR and drifted in period. As discussed in the introduction, many authors have used this SLS3 longitude system $\lambda_{\mathrm{SLS} 3}$ to study periodic phenomena in Saturn's magnetosphere. Other authors have used slightly different formalisms [e.g., Southwood and Kivelson, 2007; Andrews et al., 2008; Provan et al., 2009a], but these are ultimately derived from the time-dependent phase deduced by Kurth et al. [2008]. The phase of SKR, $\Phi_{\mathrm{SKR}}(\mathrm{t})$, can be written as

$$
\Phi_{S K R}(t)=\omega_{0} t-\Delta \Phi_{S K R}(t),
$$

where $\omega_{0}$ is the angular frequency of some reference period (equal to 0.4497 days or $10.7928 \mathrm{~h}$ ), $\Delta \Phi_{\mathrm{SKR}}(\mathrm{t})$ is the timedependent drift in phase of SKR due to the drifting period, and $t$ is the epoch time in days since the start of 1 January 2004. The SLS3 longitude system defined by Kurth et al. [2008, and references therein] can be obtained from this phase by correcting for the local time of the spacecraft and shifting by $100^{\circ}$ (so that SKR maximum occurs when the subsolar SLS3 longitude is $\left.100^{\circ}\right), \lambda_{\mathrm{SLS} 3}(\mathrm{t}, \varphi)=\Phi_{\mathrm{SKR}}(\mathrm{t})-\varphi+$ $100^{\circ}$, where $\varphi=15^{\circ}(\mathrm{LT}-12)$ is the local time azimuth of the spacecraft (measured positive from noon) and LT is the local time of the spacecraft in hours. Andrews et al. [2008] and Provan et al. [2009a] defined a magnetic guide phase $\Phi_{\mathrm{M}}(\mathrm{t})$ that is related to the SKR phase but has an almost constant phase offset $\psi_{M}$, such that $\Phi_{M}(\mathrm{t})=\Phi_{S K R}(\mathrm{t})-\psi_{M}(\mathrm{t})$. The phase factor $\psi_{\mathrm{M}}(\mathrm{t})$ is an angle that expresses the phase of the magnetic field oscillations relative to the SKR phase. Provan et al. [2009a] determined $\psi_{\mathrm{M}}$ empirically by crosscorrelating a model oscillation whose periodicity was equal to the SKR modulation period as determined by Kurth et al. [2008], with suitably processed magnetic field data from Cassini's SOI pass in 2004 to revolution (rev) 54 at the end of 2007. The period of this magnetic signal was found to vary slightly with respect to the Kurth et al. [2008] SKR period, but Andrews et al. [2008] and Provan et al. [2009a] argued that this represented a refined accuracy in the determination of some common periodicity rather than a real physical difference in the magnetic and SKR modulations.

[20] Andrews et al. [2008] and Provan et al. [2009a] defined magnetic field periodicities in the cam region as $\mathrm{B}_{\mathrm{i}}(\mathrm{t}, \varphi)=\mathrm{B}_{\mathrm{i} 0} \cos \left(\Psi_{\mathrm{Mc}}(\mathrm{t}, \varphi)-\psi_{\mathrm{i}}\right)$, where $\mathrm{B}_{\mathrm{i}}$ is the cam perturbation field for component $\mathrm{i}, \mathrm{B}_{\mathrm{i} 0}$ is the amplitude of that component, $\Psi_{\mathrm{Mc}}(\mathrm{t}, \varphi)$ is the local time corrected magnetic phase function in the core, and $\psi_{\mathrm{i}}$ is the polarization of field component $\mathrm{i}$ relative to the magnetic phase. The core magnetic phase function $\Psi_{M c}(t, \varphi)$ is defined as

$$
\Psi_{M c}(t, \varphi)=\Phi_{S K R}(t)-\varphi-\psi_{M}(t)=\Phi_{M}(t)-\varphi .
$$

In this formalism at a given time $t$, the component $B_{i}$ varies sinusoidally in local time $\varphi$ with a maximum at a local time defined by the phase factor $\psi_{\mathrm{M}}$. This phase was constructed in such a way that $\psi_{\mathrm{r}}=\psi_{\theta}=0$ and $\psi_{\varphi}=90^{\circ}$, such that the field in the core region can simply be described by $\mathrm{B}_{\mathrm{r}} \approx \mathrm{B}_{\theta} \approx$ $\cos \left(\Psi_{\mathrm{Mc}}(\mathrm{t}, \varphi)\right)$ and $\mathrm{B}_{\varphi} \approx \sin \left[\Psi_{\mathrm{Mc}}(\mathrm{t}, \varphi)\right]$. This core magnetic phase $\Psi_{\mathrm{Mc}}(\mathrm{t}, \varphi)$ is the one that will be used in this paper.

[21] We return now to the description of how periodicities in the $\mathrm{z}$ location of Saturn's magnetospheric current sheet are introduced into the bowl-shaped magnetodisc model. We have implemented this model using both the SLS3 longitudes of Kurth et al. [2008] and also the magnetic phase model and formalism presented by Andrews et al. [2008] and others. For clarity we will present the model in the framework of Andrews et al. [2008] and Provan et al. [2009a], but the model equations as a function of SLS3 longitude are included in Appendix B.

[22] We represent the displacement of Saturn's magnetospheric current sheet from the equator by $\mathrm{z}_{\mathrm{CS}}\left[\Psi_{\mathrm{PS}}(\mathrm{t}, \varphi, \rho)\right]$, where $\Psi_{\mathrm{PS}}$ is the plasma sheet phase, which is a function of time, spacecraft local time, and radial distance. Similar to the magnetic phase $\mathrm{B}_{\mathrm{i}}(\mathrm{t}, \varphi)=\mathrm{B}_{\mathrm{i} 0} \cos \left[\Psi_{\mathrm{Mc}}(\mathrm{t}, \varphi)-\psi_{\mathrm{i}}\right]$, at some time $t$ the plasma sheet is offset from the equator and 
Table 1. Variables and Parameters Used to Model the Time-Dependent Location of the Model Plasma Sheet ${ }^{\mathrm{a}}$

\begin{tabular}{|c|c|c|}
\hline Symbol & Units & Description \\
\hline $\mathrm{z}_{\mathrm{CS}}$ & $\mathrm{R}_{\mathrm{S}}$ & Displacement of the plasma sheet from the equator along $\mathrm{z}_{\mathrm{KG}}$ axis \\
\hline$\theta_{\text {SUN }}$ & deg & Angle subtended by the Sun and Saturn's magnetic dipole equator \\
\hline$\rho$ & $\mathrm{R}_{\mathrm{S}}$ & Cylindrical distance from Saturn measured in Saturn's equator \\
\hline$r_{H}$ & $\mathrm{R}_{\mathrm{S}}$ & Hinging distance for Saturn's plasma/current sheet \\
\hline$\theta_{\mathrm{TILT}}$ & deg & Tilt angle of the model plasma sheet \\
\hline$\rho_{0}$ & $\mathrm{R}_{\mathrm{S}}$ & Distance at which plasma sheet becomes tilted \\
\hline$\varphi$ & deg & Local time angle of the spacecraft $=15^{\circ}(\mathrm{LT}-12)$ \\
\hline LT & $\mathrm{h}$ & Local time of the spacecraft \\
\hline$\Phi$ & $\operatorname{deg}$ & Some exact guide phase which may be SKR or magnetic phase \\
\hline$\Phi_{\mathrm{SKR}}$ & $\operatorname{deg}$ & SKR phase (without $100^{\circ}$ offset or local time correction) \\
\hline$\Phi_{\mathrm{M}}$ & deg & Magnetic phase \\
\hline$\Delta \Phi_{\mathrm{SKR}}$ & deg & Time dependent drift in SKR phase from a fixed period \\
\hline$\omega_{0}$ & $\operatorname{deg} \mathrm{s}^{-1}$ or $\operatorname{deg} \mathrm{d}^{-1}$ & $\begin{array}{l}\text { Nominal angular frequency of Saturn with a fixed period }=2 \pi / \mathrm{P}_{0} \text { defined as } \mathrm{P}_{0} \\
=0.4497 \text { days or } 10.7928 \mathrm{~h}\end{array}$ \\
\hline$\Psi$ & deg & Phase of a particular oscillation \\
\hline$\Psi_{\mathrm{Mc}}$ & deg & Magnetic phase of oscillations in the core \\
\hline$\psi_{\mathrm{M}}$ & deg & Phase of oscillations in the cam region relative to SKR phase \\
\hline$\Psi_{\mathrm{PS}}$ & deg & Phase of model plasma sheet oscillations \\
\hline$\psi_{\mathrm{PS}}$ & deg & Phase offset (prime meridian) of plasma sheet extrema \\
\hline$\lambda$ & $\operatorname{deg}$ & Longitude within the plasma sheet \\
\hline$\lambda_{\mathrm{SLS} 3}$ & deg & SLS3 longitude \\
\hline$\lambda_{0}$ & $\operatorname{deg}$ & Prime meridian of plasma sheet extrema \\
\hline$\Omega_{\mathrm{Mc}}$ & deg & Angular frequency of magnetic modulations in the core \\
\hline$\Omega_{\mathrm{SKR}}$ & $\operatorname{deg}$ & Angular frequency of SKR modulations \\
\hline $\mathrm{V}_{\mathrm{WAVE}}$ & $\mathrm{R}_{\mathrm{S}} \mathrm{h}^{-1}$ & Propagation speed of plasma sheet oscillations \\
\hline
\end{tabular}

${ }^{\mathrm{a}}$ Arridge et al. [2008b], Kurth et al. [2008], Provan et al. [2009a], and Andrews et al. [2008].

varies sinusoidally in local time and about this offset. Both the offset and sinusoidal amplitude vary with cylindrical (used throughout) radial distance, and the phase is also a function of radial distance in order to capture any effects of "longitudinal" delay (spiral patterns). We represent the displacement of Saturn's magnetospheric current sheet with spheric periodicities was identified by Cowley et al. [2006]. The period of the signal, $\Omega_{\mathrm{Mc}}$, is calculated from Provan's phase model by taking the first derivative (with respect to time) of the phase $\mathrm{d} \Phi_{\mathrm{Mc}} / \mathrm{dt}$. The different variables and parameters are listed in Table 1 for clarity.

$z_{C S}(t, \varphi, \rho)= \begin{cases}{\left[\rho-r_{H} \tanh \left(\frac{\rho}{r_{H}}\right)\right] \tan \theta_{S U N}} & \rho<\rho_{0} \\ {\left[\rho-r_{H} \tanh \left(\frac{\rho}{r_{H}}\right)\right] \tan \theta_{S U N}+\left(\rho-\rho_{0}\right) \tan \theta_{T I L T} \cos \Psi_{P S}(t, \varphi, \rho)} & \rho \geq \rho_{0} .\end{cases}$

This modified expression is very similar to structural models of the Jovian magnetodisc [e.g., Kivelson et al., 1978; Khurana and Schwarzl, 2005]. The left-hand term on the right-hand side is essentially a DC offset term, and the righthand term is the periodic term. The plasma sheet phase is given by

$$
\Psi_{P S}(t, \varphi, \rho)=\Psi_{M c}(t, \varphi)-\psi_{P S}-\frac{\Omega_{M c}\left(\rho-\rho_{0}\right)}{v_{W A V E}} .
$$

In equation (5), $\psi_{\mathrm{PS}}$ is a prime meridian (and will be referred to as such in this manuscript), a "longitude" or phase at which the plasma sheet is at a vertical extremum. Ignoring longitudinal delays, the plasma sheet would be furthest from the equator at a phase $\Psi_{\mathrm{Mc}}=\psi_{\mathrm{PS}}$, and for a core magnetic phase $\Phi_{\mathrm{Mc}}=0^{\circ}$, the plasma sheet will be found furthest from the equator at a local time angle of $\varphi=-\psi_{\mathrm{PS}}$. The longitudinal delays, introduced by the term $\Omega_{\mathrm{Mc}}\left(\rho^{-} \rho_{0}\right) / \mathrm{V}_{\mathrm{WAVE}}$, are essentially propagation effects produced by the finite transit time for the signal to travel a distance $r$ at a speed $\mathrm{v}_{\text {WAVE. }}$ Such a phase delay in Saturn's global magneto-
[23] To obtain best fit model parameters $\left\{\mathrm{R}_{\mathrm{H}}, \rho_{0}, \theta_{\mathrm{TILT}}\right.$, $\left.\psi_{\mathrm{PS}}, \mathrm{V}_{\mathrm{WAVE}}\right\}$ (or $\left\{\mathrm{R}_{\mathrm{H}}, \rho_{0}, \theta_{\mathrm{TILT}}, \lambda_{0}, \mathrm{~V}_{\mathrm{WAVE}}\right\}$, compare Appendix B), the model must be compared with data. Given a large database of clear current sheet crossings, the rootmean-square (RMS) difference between the modeled and observed current sheet crossing locations could be minimized to obtain the model parameters. The sparse distribution of clear current sheet crossings implies that an alternative approach may constrain the model parameters in a more consistent fashion and/or may permit one to build a model that allows us to track temporal changes in the plasma sheet. This latter point is important in a system that appears to have a variable period.

[24] To fit the model to Cassini data, it is desirable to use nonlinear least squares to minimize the deviation between measured and modeled plasma sheet electron number densities. The electron density profile about the center of the plasma sheet was modeled using the thermal pressure associated with a Harris neutral sheet, assuming a constant electron temperature, as a mathematically convenient representation 

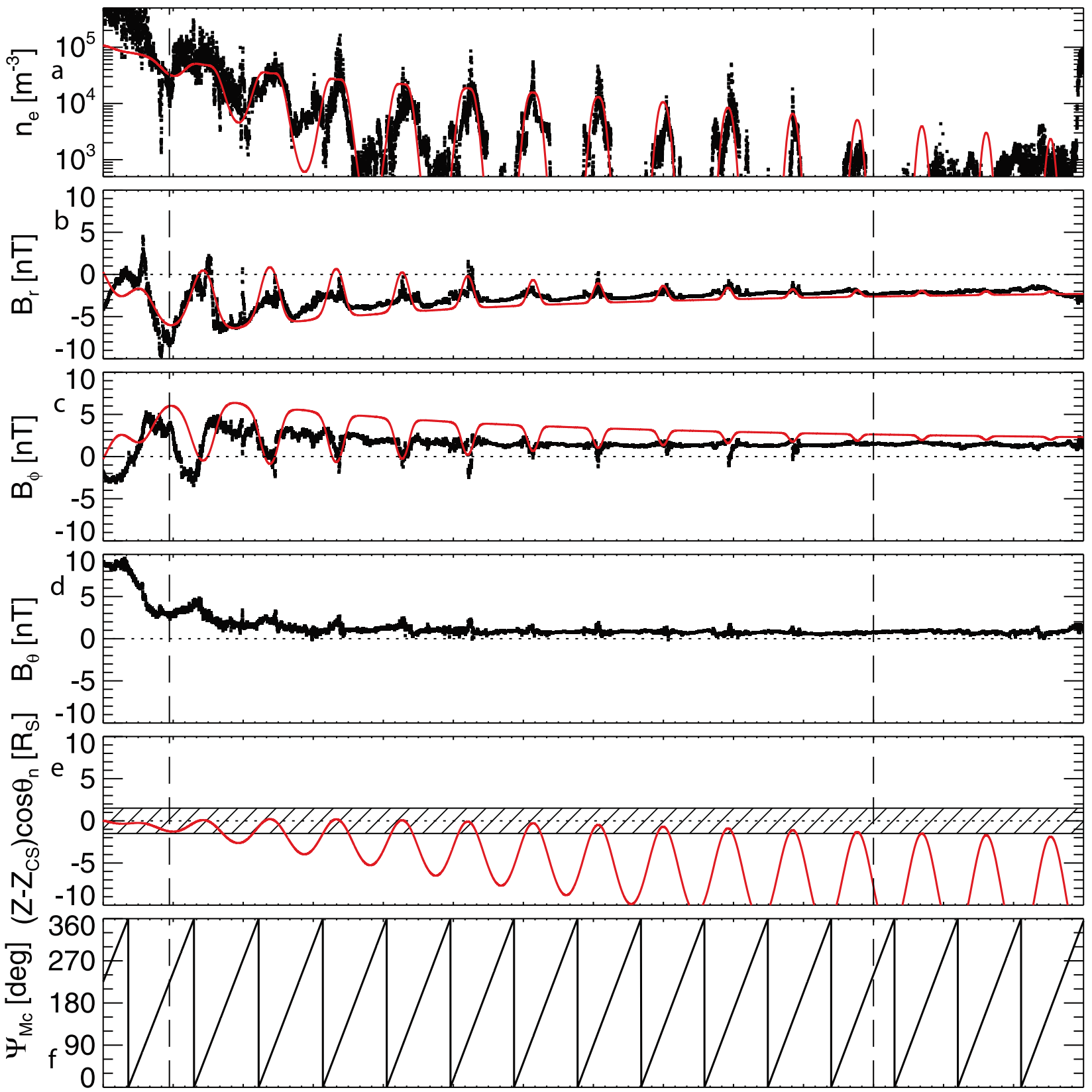

$\begin{array}{llllllllllllll}0 & 12 & 24 & 36 & 48 & 60 & 72 & 84 & 96 & 108 & 120 & 132 & 144 & 156\end{array}$

$\begin{array}{lllllllllllllll}\text { RHO } & 10.6 & 15.2 & 19.4 & 23.2 & 26.6 & 29.8 & 32.7 & 35.4 & 37.9 & 40.3 & 42.5 & 44.6 & 46.5 & 48.4\end{array}$

$\begin{array}{lllllllllllllll}\text { LAT } & 0.38 & 0.34 & 0.30 & 0.26 & 0.24 & 0.22 & 0.20 & 0.18 & 0.17 & 0.15 & 0.14 & 0.13 & 0.12 & 0.11\end{array}$

$\begin{array}{lllllllllllllll}\text { SLT } & 22.9 & 0.3 & 1.0 & 1.5 & 1.9 & 2.2 & 2.4 & 2.6 & 2.7 & 2.9 & 3.0 & 3.1 & 3.2 & 3.3\end{array}$

Figure 3. Data from the outbound leg of Cassini's rev 20 (18-24 January 2006, days 018-024) showing electron and magnetic field periodicities consistent with a flapping plasma sheet. (a) Electron number density in black with the model (equations (2)-(4)) density in red, (b and c) the spherical radial and azimuthal components of the magnetic field with the model (equations (2)-(4)) with $\mathrm{B}_{\mathrm{r}} \propto \tanh \left(\mathrm{z}^{\prime} / \mathrm{H}\right)$ and $\mathrm{B}_{\varphi} \propto-\tanh \left(\mathrm{z}^{\prime} / \mathrm{H}\right)$ profiles in red, (d) the polar (co-latitudinal) component of the magnetic field, (e) the spacecraft position (red) in a frame at rest with respect to the plasma sheet (hatched rectangle), and (f) the local time corrected magnetic phase according to the formulae given by Provan et al. [2009a]. The dashed vertical lines indicate the interval considered when fitting the model to the data. The annotation at the bottom of the plot gives (1) the number of hours from 18 January 2006, (2) the radial distance of the spacecraft from Saturn in $\mathrm{R}_{\mathrm{S}}$, (3) the latitude of the spacecraft in degrees, and (4) the local time of Cassini in hours. 
Table 2. Summary of Fitted Parameters from Cassini Passes Through Rev 20-30 Using the Provan et al. [2009a] Magnetic Phase Model $\left(\psi_{\mathrm{PS}}\right.$ Phase) and Kurth et al. [2008] SLS3 Longitude $\left(\lambda_{\mathrm{SLS} 3}\right)^{\mathrm{a}}$

\begin{tabular}{|c|c|c|c|c|c|c|c|}
\hline & \multirow[b]{2}{*}{$\psi_{\mathrm{PS}}(\mathrm{deg})$} & \multirow[b]{2}{*}{$\lambda_{\mathrm{SLS} 3}(\mathrm{deg})$} & \multirow[b]{2}{*}{$\mathrm{R}_{\mathrm{H}}\left(\mathrm{R}_{\mathrm{S}}\right)$} & \multicolumn{3}{|c|}{ Delay } & \multirow[b]{2}{*}{$\mathrm{H}\left(\mathrm{R}_{\mathrm{S}}\right.$} \\
\hline & & & & $\mathrm{R}_{\mathrm{S}}$ per Hour & $\begin{array}{l}\text { Kilometers } \\
\text { per Second }\end{array}$ & $\begin{array}{c}\text { Degrees } \\
\text { per } R_{S}\end{array}$ & \\
\hline Rev 20 outbound & $210 \pm 17$ & $121 \pm 17$ & 20 & 9 & 150 & 3.7 & 1.5 \\
\hline Rev 21 outbound & $174 \pm 12$ & $90 \pm 12$ & 22 & 7 & 120 & 4.8 & 1.5 \\
\hline Rev 22 outbound & $212 \pm 19$ & $130 \pm 19$ & 22 & 8 & 135 & 4.3 & 1.5 \\
\hline Rev 24 outbound & $171 \pm 17$ & $95 \pm 17$ & 20 & 7 & 120 & 4.8 & 3 \\
\hline Rev 25 outbound & $146 \pm 31$ & $71 \pm 31$ & 16 & 5 & 84 & 6.7 & 3 \\
\hline Rev 26 inbound & $251 \pm 23$ & $176 \pm 23$ & 20 & 13 & 220 & 2.6 & 2 \\
\hline Rev 26 outbound & $125 \pm 24$ & $50 \pm 24$ & 30 & 13 & 220 & 2.6 & 6 \\
\hline Rev 27 inbound & $259 \pm 17$ & $184 \pm 17$ & 16 & 13 & 220 & 2.6 & 1.5 \\
\hline Rev 28 inbound & $278 \pm 14$ & $202 \pm 14$ & 32 & 10 & 170 & 3.3 & 1.5 \\
\hline Rev 29 inbound & $240 \pm 13$ & $163 \pm 13$ & 30 & 16 & 270 & 2.1 & 2 \\
\hline Rev 30 inbound & $272 \pm 16$ & $193 \pm 16$ & 18 & 10 & 170 & 3.3 & 3 \\
\hline
\end{tabular}

${ }^{\mathrm{a}}$ The magnetic phases were converted to SLS3 longitudes using $\lambda_{\mathrm{SLS} 3}(\mathrm{t}, \varphi)=\Psi_{\mathrm{Mc}}(\mathrm{t}, \varphi)+\psi_{\mathrm{M}}(\mathrm{t})+100^{\circ}$.

for the distribution of plasma about the center of the plasma sheet (justified further in Appendix C):

$$
n=n_{0} \operatorname{sech}^{2}\left(\frac{z^{\prime}}{H}\right)
$$

Here $\mathrm{z}^{\prime}=\left(\mathrm{z}_{\mathrm{SC}}-\mathrm{z}_{\mathrm{CS}}\right) \cos \theta_{\mathrm{n}}$ is obtained from the structural model (equations (4) and (5)) and is the distance from the spacecraft to the plasma sheet center, along the local normal to the plasma sheet. The angle $\theta_{\mathrm{n}}$ is the angle between the $z$ axis and the sheet normal vector. This vector can be calculated straightforwardly from the cross product of two vectors in the plane of the current sheet, which can be found from the partial derivatives of $\mathrm{z}_{\mathrm{CS}}$ with respect to $\mathrm{r}$ and $\varphi$, and were calculated analytically in this work; see Arridge et al. [2008b, paragraph 32] for more details, but where their calculation is modified to include the tilt due to the second term in equation (4).

[25] The remaining quantity in equation $(6), \mathrm{n}_{0}(\mathrm{r})$, is the central plasma sheet density that is a function of radial distance $r$. One could prescribe a functional dependence for $\mathrm{n}_{0}(\mathrm{r})$ and also fit for the free parameters of $\mathrm{n}_{0}(\mathrm{r})$. However, there is an interdependence between the hinging of the plasma sheet and the density profile in the plasma sheet that introduces a highly nonlinear interaction between the parameters of $n_{0}(r)$ and the other free parameters of the model. At a given spacecraft position, the model plasma sheet density is dependent on the location of the plasma sheet and the central plasma sheet density. If the density at a given spacecraft location is too small compared with the data, the nonlinear minimizer can either move the plasma sheet closer to the spacecraft (change $\mathrm{R}_{\mathrm{H}}$ ), or increase the central plasma sheet density (change $\mathrm{n}_{0}(\mathrm{r})$ ). Since both $\mathrm{z}^{\prime}$ and $\mathrm{n}_{0}$ are functions of $r$, it is difficult for the minimizer to correctly fit for both quantities simultaneously. To solve this issue, a model for the variation of the central plasma sheet density, $\mathrm{n}_{0}(\mathrm{r})=$ $\left(5.62 \times 10^{6}\right) \mathrm{r}^{-1.67}$, was derived using survey results presented by Arridge et al. [2009]. The details of this simple model, valid between 15 and $70 \mathrm{R}_{\mathrm{S}}$, are presented in Appendix A.

[26] Equations (4)-(6) were used to construct an unweighted $\chi^{2}$ merit function (7) that was minimized using the Levenberg-Marquadt algorithm [Press et al., 1992]:

$$
\chi^{2}=\sum_{i}\left[n_{i}-n_{0}\left(r_{i}\right) \operatorname{sech}^{2}\left(\frac{\left(z_{S C, i}-z_{C S, i}\right) \cos \theta_{n, i}}{H}\right)\right]^{2} .
$$

For most of the intervals studied, the minimizer often found local minima that did not correspond to physically meaningful results. For all but one of the data sets considered in this paper it was also found that the minimizer would not converge when the prime meridian $\psi_{\text {PS }}$ was included as a free parameter. Hence it was not possible to obtain the prime meridian by a direct fit to the data. The electron densities used in this study were $32 \mathrm{~s}$ averages and at this cadence there were relatively few measurements near the center of the plasma sheet, due to rapid motion of the plasma sheet. Hence the minimization is naturally weighted toward accurately modeling the lower density outer plasma sheet. This work does not require a detailed modeling of the plasma sheet but it is important to accurately reproduce the timing of plasma sheet encounters and crossings and to be able to distinguish between the two. Using these requirements, it was decided to manually fit the model to the data by manipulating the free parameters. In principle one could weight the data in order to place a bias toward the center of the plasma sheet, or resample the data to provide uniform sampling with distance from the center of the plasma sheet, but this remains as a topic for future work.

[27] To manually fit the model to the data, the following procedure was followed in each case. To reduce the parameter space and remove nonlinearities, the tilted inner edge $\rho_{0}$ and the tilt angle $\theta_{\text {TILT }}$ were fixed at $12 \mathrm{R}_{\mathrm{S}}$ and $12^{\circ}$, respectively. These values were selected from an initial investigation into the periodicities on the outbound pass of (rev) 20 , where the inner edge was found to lie at $12 \mathrm{R}_{\mathrm{S}}$ but with a smaller tilt angle of $6^{\circ}$. Larger tilt angles were found to generate more pronounced density minima, which were in better agreement with the data (this is discussed in more detail in section 4.1; see also Figure 3). These tilt angles are consistent with "dipole tilts" of around $10^{\circ}$ estimated by previous studies [e.g., Provan et al., 2009a; K. K. Khurana, private communication, 2006].

[28] The remaining parameters to be fitted were the prime meridian, hinging distance, and wave speed. The prime meridian was adjusted in $5^{\circ}$ steps in order to match the density maxima near $15 \mathrm{R}_{\mathrm{S}}$. The timings of the more distant plasma sheet encounters were matched by manipulating the wave speed in steps of $1 \mathrm{R}_{\mathrm{S}} \mathrm{h}^{-1}$, and the hinging distance was fixed by matching the heights of the density maxima as a function of radial distance. Often the wave speed and hinging distance were adjusted in an iterative fashion to 


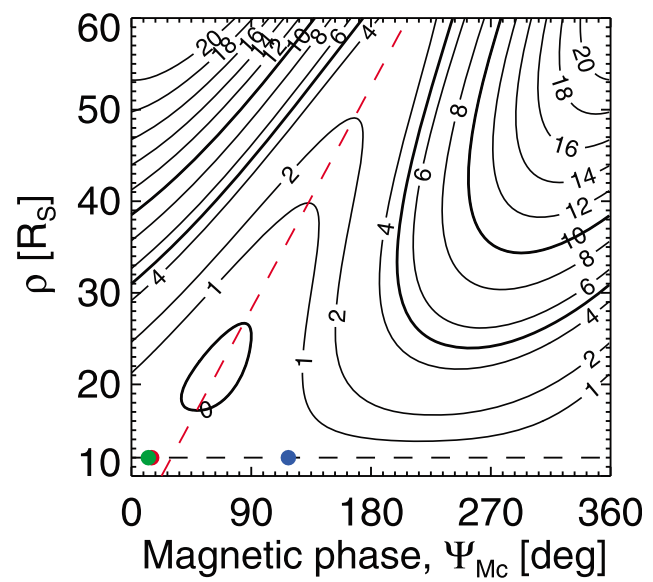

Figure 4. Contour plot showing the displacement of the plasma sheet from the equatorial plane as a function of magnetic phase and radial distance for the parameters obtained from the rev 20 outbound pass (Figure 3). The bold contours indicate $\mathrm{z}_{\mathrm{CS}}=0,5$, and $10 \mathrm{R}_{\mathrm{S}}$. The wave-delayed phase $\psi_{\mathrm{PS}}+\Omega_{\mathrm{Mc}}\left(\rho-\rho_{0}\right) / \mathrm{v}_{\mathrm{WAVE}}$ is indicated by the red dashed line. The colored circles represent the phase of the core magnetic oscillations, shifted in phase to $12 \mathrm{R}_{\mathrm{S}}$ using the delays presented by Andrews et al. [2010a] appropriate for the midnight and post-midnight sector. Here we use the color-coding scheme of Andrews et al. [2008] where red represents $\mathrm{B}_{\mathrm{r}}$, green represents $\mathrm{B}_{\theta}$, and blue represents $\mathrm{B}_{\varphi}$. The plasma sheet extrema are thus in phase with the core $\mathrm{B}_{\mathrm{r}}$ and $\mathrm{B}_{\theta}$ perturbations.

produce the best visual agreement. For some passes the plasma sheet thickness was adjusted from a nominal $1.5 \mathrm{R}_{\mathrm{S}}$ [Kellett et al., 2009; N. Sergis, private communication, 2011] to provide the best agreement with the crossings of the current sheet. Occasionally, changes to the wave speed required slight modifications to the prime meridian; once such a change was made the wave speed and hinging distance were iteratively updated. Empirically it was also found that often very little difference could be discerned between adjacent $10^{\circ}$ or even $20^{\circ}$ steps.

[29] To provide some quantitative understanding of this variability in $\psi_{\mathrm{PS}}$, these steps were carried out five times for each pass. To ensure that each fit was independent, a random constant factor was added to the magnetic phase for each of the five fits and then removed once the prime meridian had been found. The quoted phases are the mean of the results and the error is the range. Very little change could be detected in the hinging distance or wave speed and so these were fixed for each set of five. The errors were typically around $20^{\circ}$, which is slightly larger than the $10^{\circ}$ uncertainty in the phase model itself [Kurth et al., 2008], but which is comparable to the phase "jitter" reported and discussed by Andrews et al. [2008] and Provan et al. [2011]. From these considerations we conservatively estimate an uncertainty in the phase of $20^{\circ}$. Finally, because the model has been fitted to electron number densities, there can be an inherent ambiguity of $180^{\circ}$ in the phase of the fitted model. To solve this issue, the structural model was also compared with the radial and azimuthal components of the magnetic field using a corresponding Harris model for the magnetic field: $\mathrm{B}_{\rho}\left(\mathrm{z}^{\prime}\right)=\mathrm{B}_{\text {lobe }} \tanh \left(\mathrm{z}^{\prime} / \mathrm{H}\right)$ and $\mathrm{B}_{\varphi}\left(\mathrm{z}^{\prime}\right)=$
$-\mathrm{B}_{\text {lobe }} \tanh \left(\mathrm{z}^{\prime} / \mathrm{H}\right)$, where $\mathrm{B}_{\text {lobe }}$ is a radially dependent lobe magnetic field strength model [Jackman and Arridge, 2011].

[30] The inbound and outbound legs of Cassini on revs 20-30 were analyzed in this fashion, but the data for 10 out of 22 passes had significant data gaps or the periodicities were not particularly evident. The outbound pass of rev 27 occurred during the passage of a CIR that produced considerable dynamics in the location of the plasma sheet (N. André et al., manuscript in preparation, 2011) and so we did not analyze this pass (although see the discussion in section 4.3). Table 2 presents the modeling results for all 10 passes, using the phase models of Provan et al. [2009a] and Kurth et al. [2008], where a fit could be made to the electron number densities. These results are also summarized in Figure 10 using the Provan et al. [2009a] phase model. In section 4, the results of the analysis will be discussed for a number of tail passes that illustrate the range of signatures and parameter values found in this study.

\section{Analysis of Selected Tail Passes}

\subsection{Midnight/Pre-dawn Equatorial Passes}

[31] Figure 3 presents the results from fitting the model in section 3 to data from the outbound portion of Cassini's rev 20 during 18-24 January (days 018-024) 2006. During this part of the orbit Cassini moved from 2300 to $0300 \mathrm{~h}$ local time and from 11-45 $R_{S}$ in radial distance. Cassini was close to the equatorial plane during the whole interval.

[32] This pass of Cassini proved to be one of the few that were tractable to a nonlinear least squares fit. Applying the nonlinear technique described in section 3 provided the following best fit parameters: $\mathrm{R}_{\mathrm{H}}=28.6 \pm 0.8 \mathrm{R}_{\mathrm{S}}, \rho_{0}=12 \pm$ $2 \mathrm{R}_{\mathrm{S}}, \theta_{\mathrm{TILT}}=6.1 \pm 0.9^{\circ}, \mathrm{v}_{\mathrm{WAVE}}=15.6 \pm 0.5 \mathrm{R}_{\mathrm{S}} \mathrm{h}^{-1}$, and $\psi_{\mathrm{PS}}=222 \pm 23^{\circ}$. To be consistent with the other results in this paper, this pass was also fitted using the manual technique that yielded results that were broadly consistent with the above nonlinear least squares values. However, the fitted tilt angle of $6.1 \pm 0.9^{\circ}$ differs considerably from that assumed in the manual fitting. During the analysis of the results of the nonlinear fit, it was found that deeper lobe density minima (which produce a better agreement with the data) could be obtained by increasing the tilt angle to $12^{\circ}$ with almost no change in the other parameters. The manually fitted results are presented in Table 2 and in Figure 3.

[33] From Figure 3 one can see that the model represents a reasonable fit to the data and accurately captures the entries into the plasma sheet. With only minor modifications, principally to the hinging distance and wave speed, these parameters were found to produce good model fits to the data from the outbound segments of revs 21 and 22, which followed similar trajectories. Orbit-to-orbit variability in the hinging distance can be interpreted in terms of variations in solar wind dynamic pressure or changes in mass content of the plasma sheet: Increases in dynamic pressure or a decrease in the mass of the plasma sheet can displace the plasma sheet further from the equator. The abrupt end to the pattern of the periodicities at $128 \mathrm{~h}$ (just before the dashed vertical marker) is coincident with a slight increase in the lobe field strength (using the radial field as a proxy for $|\mathbf{B}|)$. This is consistent with the idea of a slight increase in solar wind dynamic pressure compressing the magnetotail, increasing the lobe field strength, and simultaneously 

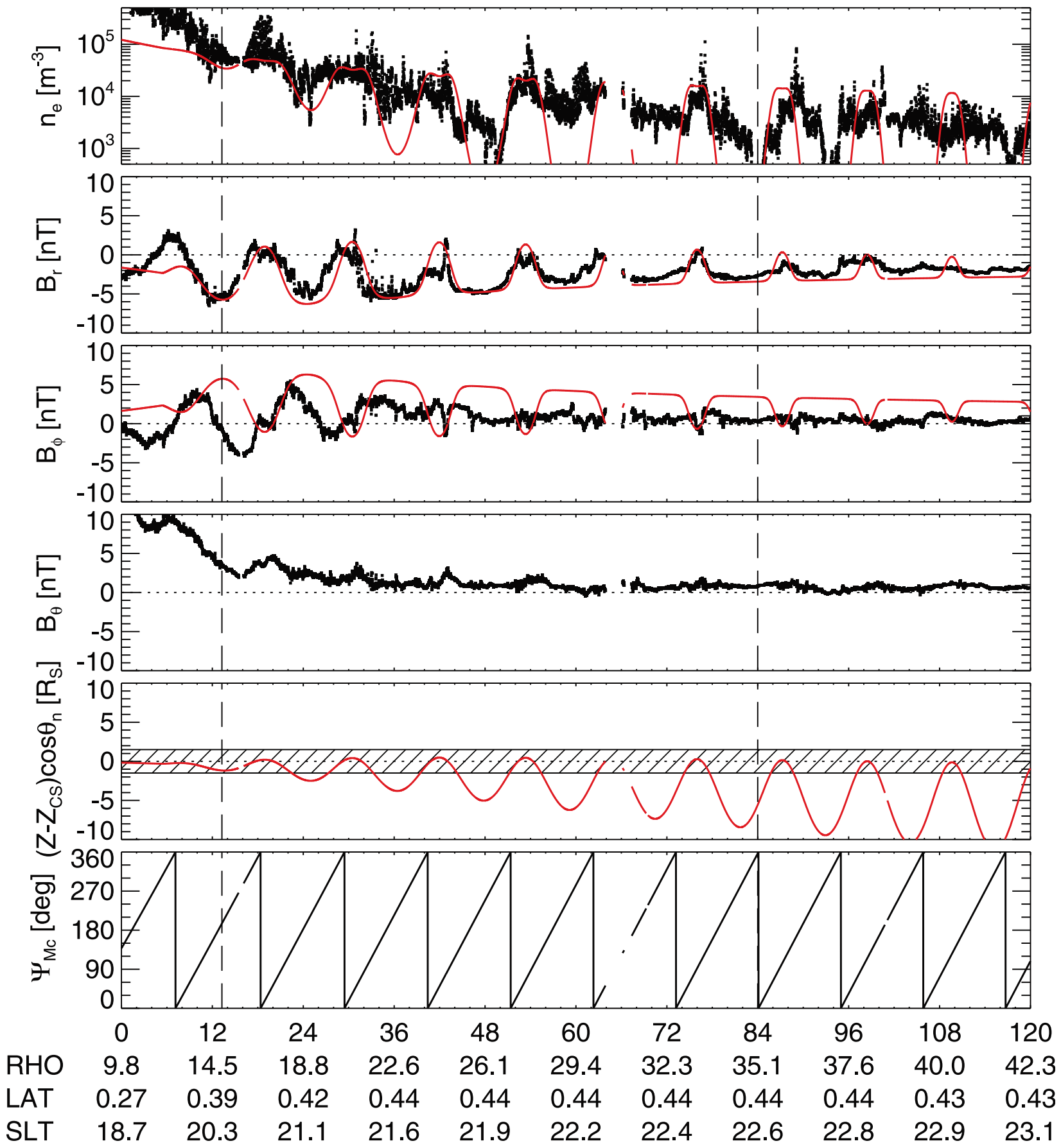

Figure 5. Data from the outbound leg of Cassini's rev 24 (23-27 May 2006, days 143-147) showing electron and magnetic field periodicities consistent with a flapping plasma sheet. Same as Figure 3 except the hours annotation is given from the beginning of 23 May 2006.

displacing the plasma sheet in a northerly direction, although detailed study and searching for solar wind evidence of this scenario is beyond the scope of this paper. For sufficiently large displacements the flapping plasma sheet may no longer encounter the spacecraft and thus provides an explanation for the apparent disappearance of the periodicities beyond $128 \mathrm{~h}$.

[34] The fitted wave speed of $9 \mathrm{R}_{\mathrm{S}} \mathrm{h}^{-1}\left(=150 \mathrm{~km} \mathrm{~s}^{-1}\right)$ is consistent with a phase delay of $3.7^{\circ} \mathrm{R}_{\mathrm{S}}^{-1}$ and is in good agreement with the findings of Carbary et al. [2007b], who found phase delays between 2.7 and $4.7^{\circ} R_{S}^{-1}$. The wave speed is approximately a factor of three larger than the wave speeds presented by Cowley et al. [2006] $\left(\sim 50 \mathrm{~km} \mathrm{~s}^{-1}\right)$ although these calculations were based on Voyager estimates for the bulk plasma parameters. Using bulk plasma parameters estimated from Cassini CAPS data [e.g., Thomsen et al., 2010], one finds Alfvén wave and sound speeds more compatible with these fitted wave speeds.

[35] The fitted prime meridians for revolutions 20, 21, and 22 were found to range between $174-212^{\circ}$ in the magnetic 

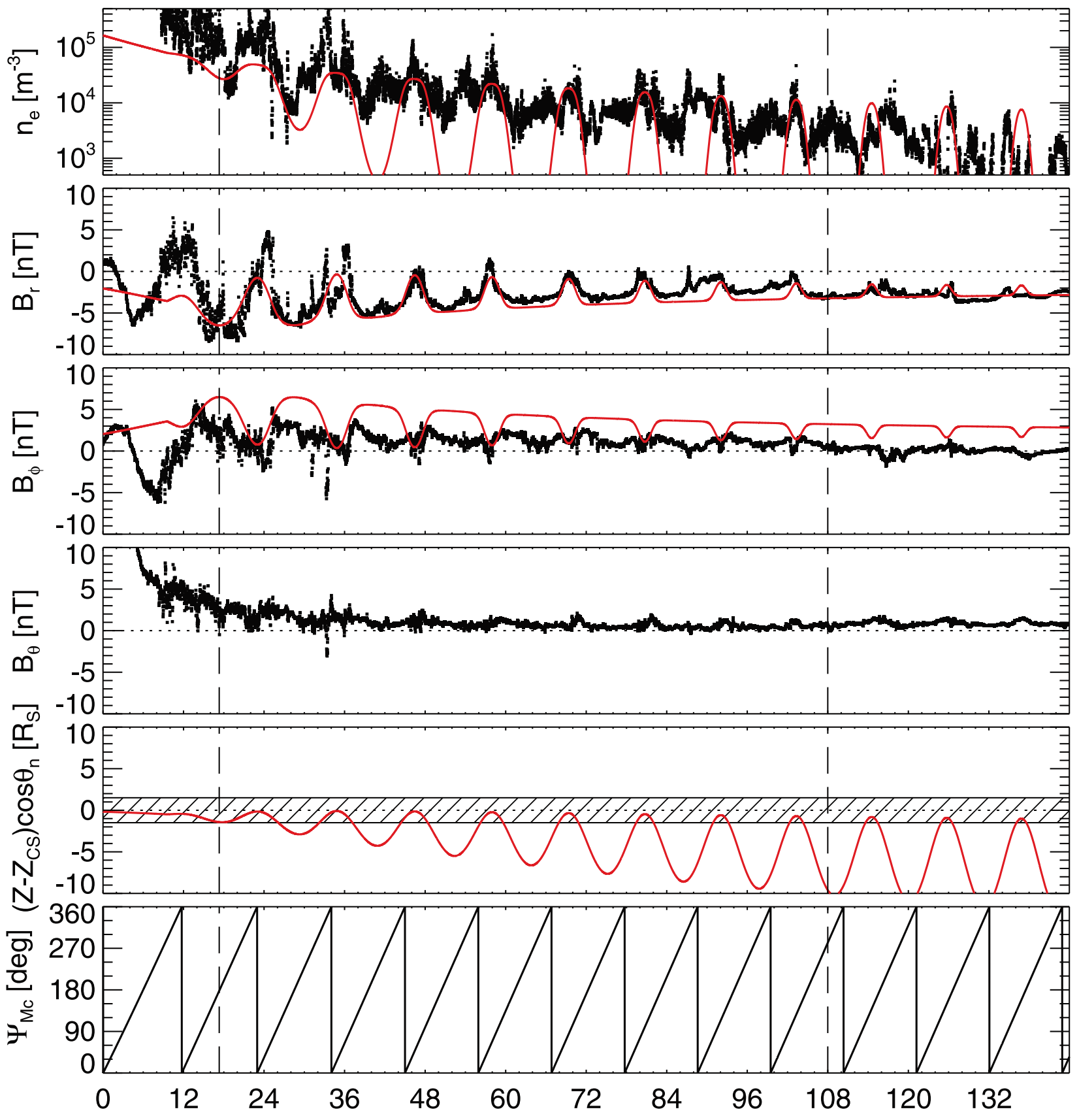

108

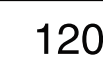

132

$\begin{array}{lcccccccccccc}\text { RHO } & 8.2 & 13.0 & 17.4 & 21.4 & 24.8 & 27.7 & 30.4 & 32.8 & 35.0 & 36.9 & 38.7 & 40.3 \\ \text { LAT } & 0.10 & 0.28 & 0.34 & 0.37 & 0.39 & 0.41 & 0.42 & 0.42 & 0.43 & 0.43 & 0.44 & 0.44 \\ \text { SLT } & 17.7 & 19.8 & 20.8 & 21.3 & 21.7 & 21.9 & 22.1 & 22.3 & 22.5 & 22.6 & 22.7 & 22.9\end{array}$

Figure 6. Data from the outbound leg of Cassini's rev 25 (1-6 July 2006, days 182-187) showing electron and magnetic field periodicities consistent with a flapping plasma sheet. Same as Figure 3 except the hours annotation is given from the beginning of 1 July 2006.

phase system and $90-130^{\circ}$ in SLS3 longitude. This is the phase at which the plasma sheet is the furthest from the equator, and hence an observer at the equator should see a minimum in plasma density. This is in approximate agreement with the findings of Arridge et al. [2008a], who observed a minimum (from data around $20 \mathrm{R}_{\mathrm{S}}$ ) in plasma density, and hence a maximum displacement of the plasma sheet from the equator at $\lambda_{\mathrm{SLS} 3}=170 \pm 20^{\circ}$ (using an average delay of $\sim 4^{\circ} \mathrm{R}_{\mathrm{S}}^{-1}$, the delay from $12-20 \mathrm{R}_{\mathrm{S}}$ is $32^{\circ}$, thus shifting the prime meridian to $122-162^{\circ}$ SLS3 longitude).

[36] The relationship between the prime meridian, longitudinal delay, and the location of the plasma sheet is illustrated visually in Figure 4. Figure 4 shows the vertical distance of the plasma sheet from the equator as a function of magnetic phase and radial distance and conveys the same information as Figure 2 of Carbary et al. [2007b]. In this 


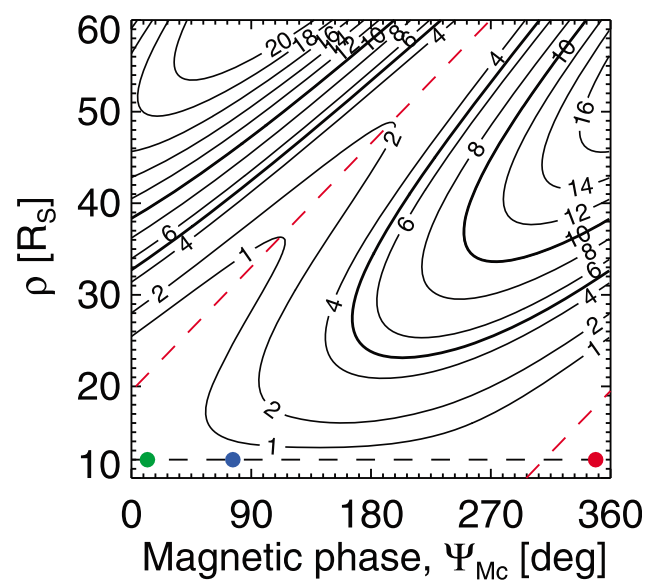

Figure 7. Contour plot showing the displacement of the plasma sheet from the equatorial plane as a function of magnetic phase and radial distance in the same format as Figure 4 but for the parameters obtained from the rev 25 outbound pass. As in Figure 4, the colored circles represent the phase of the core magnetic oscillations, shifted in phase to $12 \mathrm{R}_{\mathrm{S}}$ using the delays presented by Andrews et al. [2010a]. However, in this case the delays have been taken from the dusk and post-dusk sector to match the trajectory of the outbound rev 25 pass.

plot an observer in the equatorial plane will find density maxima along the red dashed line, where the contours indicate the plasma sheet is closest to the equator. The colored dots in Figure 4 represent the phase of magnetic oscillations in the cam (core) region that have been shifted in phase to $12 \mathrm{R}_{\mathrm{S}}$ using the delays presented by Andrews et al. [2010a]. The plasma sheet extrema are thus in phase with the core $\mathrm{B}_{\mathrm{r}}$ and $\mathrm{B}_{\theta}$ perturbations, and not the $\mathrm{B}_{\varphi}$ core field. The details of the phase relationships between the cam field and plasma sheet oscillations will be discussed in detail in section 5.

[37] One particular anomaly is that while the $\mathrm{B}_{\mathrm{r}}$ magnetic field component and plasma densities are consistent with vertical motion of the plasma sheet, the $\mathrm{B}_{\varphi}$ component exhibits a phase behavior that is somewhat decoupled from purely vertical plasma sheet motion. However, this is not inconsistent with the observed radial gradients in the phase of $\mathbf{B}$ [Andrews et al., 2010a] as the spacecraft passes through the shell of field lines near $15 R_{S}$ that are associated with the field-aligned currents that generate the core field.

\subsection{Dusk/Pre-midnight Equatorial Passes}

[38] Figures 5 and 6 present results from fitting the model to data from the outbound legs of revs 24 and 25 during 2327 May (days 143-147) and 01-06 July (days 182-187), respectively. During these periods Cassini moved from around 2000 to $2300 \mathrm{~h}$ local time, and from 15-35 and $40 \mathrm{R}_{\mathrm{S}}$ respectively. Similar to revs 20-23, Cassini was close to the equatorial plane through both of these intervals. The principal difference between these passes and those discussed in section 4.1 is the local time of the spacecraft, particularly the local time when the spacecraft was near $15 \mathrm{R}_{\mathrm{S}}$. Once again the model is a reasonable fit to the data, with the rev 25 interval representing the best agreement with the density data. In each case the model is in much better agreement with the magnetometer data, whereas the density data are much more variable. The hinging distance and wave delays were found to be 20 and $7 \mathrm{R}_{\mathrm{S}} \mathrm{h}^{-\mathrm{P}}$ for rev 24 and $16 \mathrm{R}_{\mathrm{S}}$ and $5 \mathrm{R}_{\mathrm{S}} \mathrm{h}^{-1}$ for rev 25 and so are not too dissimilar to the values found for revs 20-22. Similar to the results for rev 20 in Figure 3, the $\mathrm{B}_{\varphi}$ magnetic field component during revs 24 and 25 does not have an anti-phase relationship with $B_{r}$ until around $20 R_{S}$ once the spacecraft has fully left the cam region.

[39] However, in contrast with the passes discussed in section 4.1 , the modeled prime meridians of $171^{\circ}$ and $146^{\circ}$ for the outbound passes of rev 24 and 25, respectively, are significantly different to those from revs 20 and 22 (rev 21 is similar to rev 24). These modeled plasma sheet phases are shifted by 27 and $52^{\circ}$, respectively, from the mean phase found on revolutions 20-22. These shifts are larger than the estimated uncertainty of $\pm 20^{\circ}$ in the plasma sheet phase. In a study of the core (cam) field, Andrews et al. [2008] found that the phase could sometimes exhibit random phase shifts, referred to as "jitter," of up to $20^{\circ}$. One could consider the observed shift on rev 24 in terms of such jitter combined with the plasma sheet phase uncertainty, but a shift of $52^{\circ}$ was found on rev 25 and interpretation in terms of jitter and phase uncertainty is not so credible and so we must search for a different interpretation. Such a phase shift was also reported by Carbary et al. [2007b, Figure 3], but their shifts were only inferred in data beyond day 201 of 2006, whereas the rev 25 results reported here have been obtained in data from day 182 onward. Figure 7 illustrates contours of the current sheet location, in a similar format to Figure 4, and highlights the shift in the prime meridian; for example, compare the intersection of the red dashed line with the horizontal dashed line between Figure 7 and Figure 4. If one assumes that the plasma sheet extrema are in phase with the core $\mathrm{B}_{\mathrm{r}}$ component, then part of the modeled plasma sheet phase shift can be explained in terms of local time asymmetries in the phase of the core field [Andrews et al., 2010a], Figure 7 clearly shows that there is still a substantial phase shift of around $40^{\circ}$, which may be accounted for by uncertainties in the magnetic and plasma sheet phases.

[40] To remind the reader, the only significant difference in spacecraft trajectory between revs $24 / 25$ and $20-22$ is the local time of the spacecraft, with minor changes to the Kronographic latitude of the spacecraft. This suggests that the abrupt shift in prime meridian is perhaps due to a local time asymmetry, but the possibility remains that an abrupt shift or a "clock reset" in the process generating the periodicities might be responsible for this prime meridian shift. However, we note that no such clock reset was found in a study of the core region magnetic data by Provan et al. [2009a]. This can be checked by finding a pass sometime after revolutions 24 and 25 that has a different local time, but which is presumably in a new "clock setting."

\subsection{Dusk/Pre-midnight Non-equatorial Pass: Rev 26 Outbound}

[41] Figure 8 presents the results from fitting the model to data from the outbound portion of Cassini's rev 26 during 25-30 July (days 206-211) 2006. The trajectory in local time and radial distance was fairly similar to that of revs 24 and 25 discussed in section 4.2; however, this orbit took Cassini out of the equatorial plane to $12^{\circ}-15^{\circ}$ Kronographic 

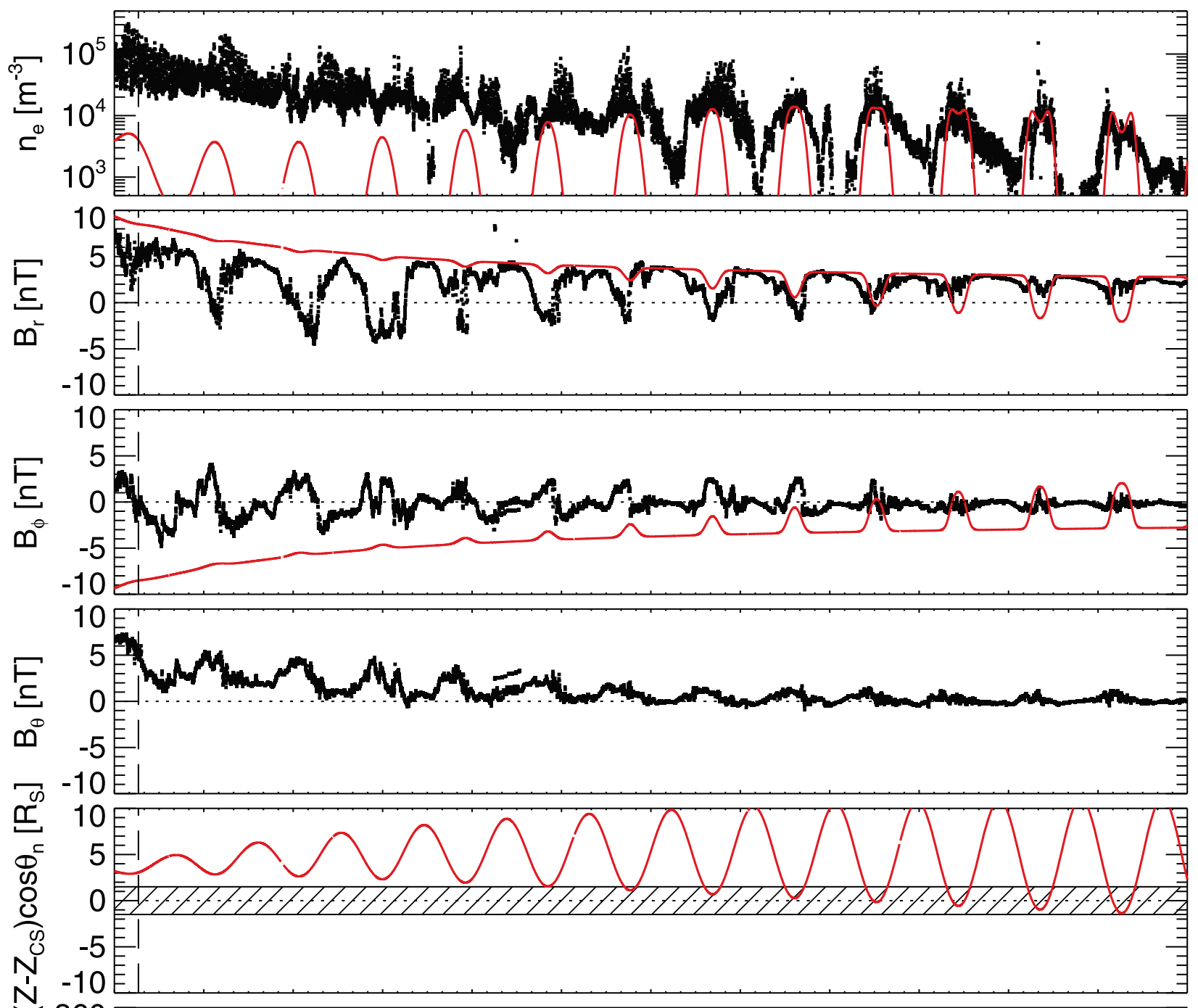

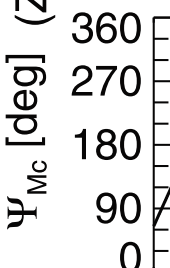

$$
0
$$
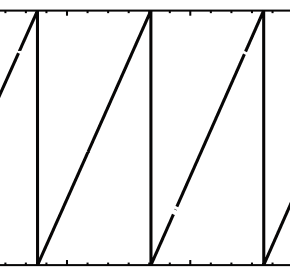

12
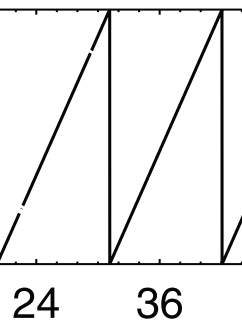

$\mathrm{RHO}$

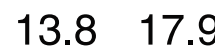

$21.5 \quad 24.7$
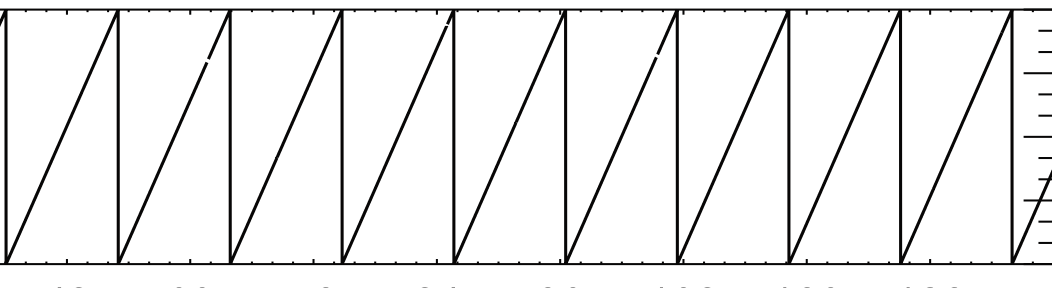

LAT

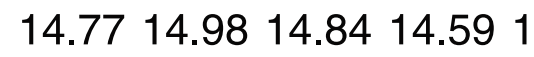

$\begin{array}{lll}48 & 60 & 72\end{array}$

84

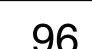

108

120

132

\section{$\begin{array}{lllllllllllll}\text { SLT } & 19.8 & 20.6 & 21.1 & 21.4 & 21.7 & 21.9 & 22.1 & 22.2 & 22.4 & 22.5 & 22.6 & 22.8\end{array}$}

Figure 8. Data from the outbound leg of Cassini's rev 26 (25-30 July 2006, days 206-211) showing electron and magnetic field periodicities consistent with a flapping plasma sheet. Same as Figure 3 except the hours annotation is given from 25 July 2006.

latitude. It is clear that the data from this pass are poorly represented by the model compared to the passes discussed thus far. Large values of both the hinging distance and plasma sheet thickness were required to obtain a reasonable agreement, although this is still not ideal and does not capture many of the current sheet crossings and encounters in the data. This shows that the flapping plasma sheet model does not provide a good representation of these data. Near
$20 \mathrm{R}_{\mathrm{S}}$ the comparison with the model suggests that the spacecraft should be closer to the center of the plasma sheet than the model predicts, but at larger Kronocentric distances the spacecraft-plasma sheet distance is approximately correct. The prime meridian of the model current sheet was also found to be shifted by $74^{\circ}-125^{\circ}$ from the $199^{\circ}$ mean on revs $20-22$.

[42] Local time asymmetries and solar wind-driven modulations of the magnetosphere may play a strong role in these 

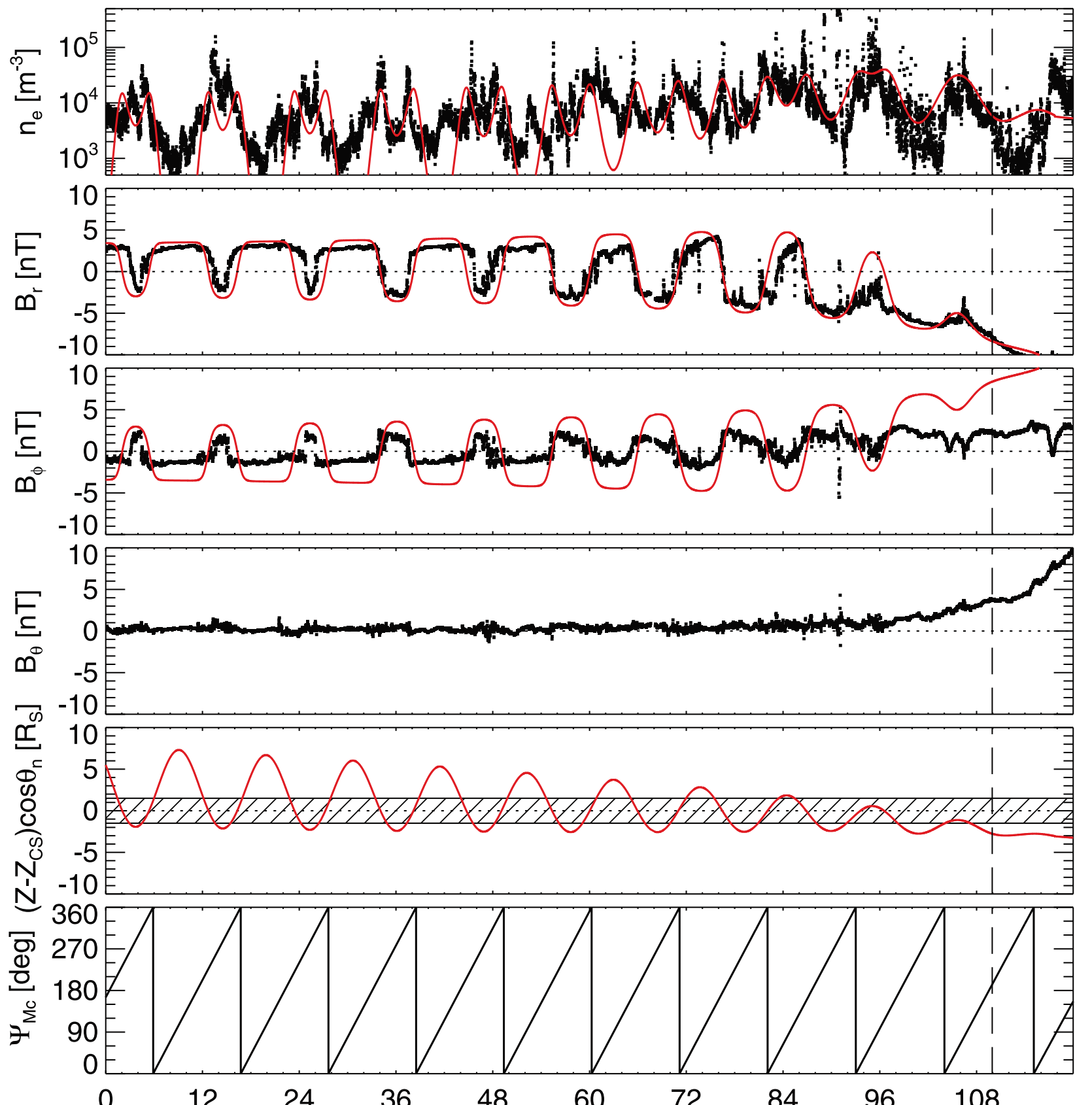

$\begin{array}{lcccccccccc}\text { RHO } & 35.1 & 34.1 & 32.9 & 31.5 & 29.8 & 27.8 & 25.4 & 22.7 & 19.4 & 15.7 \\ \text { LAT } & 9.36 & 8.60 & 7.77 & 6.86 & 5.84 & 4.67 & 3.28 & 1.56 & -1.45 & -6.88 \\ \text { SLT } & 0.9 & 1.1 & 1.2 & 1.3 & 1.5 & 1.7 & 1.9 & 2.1 & 2.4 & 2.9\end{array}$

Figure 9. Data from the inbound leg of Cassini's rev 29 (20-24 September 2006, days 263-267) showing electron and magnetic field periodicities consistent with a flapping plasma sheet. Same as Figure 3 except the hours annotation is given from 20 September 2006.

differences. In the case of the former there may be local time asymmetries in the plasma sheet density, thickness, hinging distance, and tilt angle $\theta_{\mathrm{TILT}}$, which are all difficult to account for with the available data and understanding of local time asymmetries in Saturn's magnetosphere.

[43] It should be pointed out that rev 26 outbound was at a moderately high northern latitude of around $14^{\circ}$ and so may be influenced by the different period of the northern SKR emissions and their associated magnetic perturbations [e.g., Andrews et al., 2010b]. It is particularly interesting to note that the agreement between the model and the data improves as the spacecraft moves to lower latitudes of $\sim 12^{\circ}$ toward the end of the interval. Such an effect was also observed on rev 27 outbound, although the analysis of this interval is complicated by the presence of a significant distortion of the magnetosphere (André et al., manuscript in preparation, 
2011); hence, we do not show these data or include the fits in our results table. Further analysis of the high latitude passes confirms the influence of magnetic perturbations from the northern hemisphere auroral zones (G. Provan et al., Dual periodicities in planetary period magnetic field oscillations in Saturn's tail, submitted to Journal of Geophysical Research, 2011). Hence, the phase of the plasma sheet we derive from the rev 26 outbound pass is essentially meaningless in attempting to establish a phase relationship between the motion of the plasma sheet and the core magnetic perturbations that are essentially in phase with the signal from the southern auroral zone. The reader is referred to Provan et al. (submitted manuscript, 2011) for more details on the interaction between northern and southern hemisphere periods and their effects on the plasma sheet location and thickness.

\subsection{Inbound Post-midnight Non-equatorial Pass: Rev 29 Inbound}

[44] Figure 9 presents the final example discussed in this paper and shows results from fitting the model to the inbound portion of Cassini's rev 29 during 20-24 September (days 263-267) 2006. During this interval Cassini was entirely in the post-midnight sector and moved from $9^{\circ}$ above the equator to end below the equator at a latitude of $-7^{\circ}$.

[45] Double-peaked crossings of the current/plasma sheet are clear in the electron number density maxima, and reversals in the radial magnetic field component are observed due to the proximity of Cassini to the mean location of the plasma sheet [e.g., Waldrop et al., 2005]. The modeled profiles are in reasonable agreement with the data. Minor differences in the depth of entries into the plasma sheet and timing differences in the current sheet crossings may be related to transient variations in the hinging distance or modulations of the plasma sheet thickness, or might be related to "jitter" in the phase of the periodicity as observed in inner magnetospheric magnetic field periodicities [e.g., Andrews et al., 2008; Provan et al., 2011].

[46] The local time coverage of this pass provides an opportunity to test between a long-term stable shift in the prime meridian and some local time asymmetry, as discussed in section 4.2. The fitted prime meridian was $240^{\circ}$, which was very similar to that found for early revs in 2006 (e.g., section 4.1), taking into account possible jitter and the uncertainty on the prime meridian; hence, this does not show the same shift as seen on the outbound segments of revs 24-25, when Cassini was near dusk. The wide range in latitude also argues against influences from the different period of SKR and magnetic perturbations in the northern hemisphere that might influence a system apparently dominated by physical processes associated with current systems in the southern hemisphere.

\section{Discussion}

[47] In the introduction to this paper it was emphasized that the latitude of the spacecraft was important when inferring the prime meridian of a flapping plasma sheet based on plasma fluxes and/or densities. It was shown that very different prime meridian longitudes could be inferred depending on the location with respect to the sheet. In this work, periodicities in low energy electrons $(0.5 \mathrm{eV}-26 \mathrm{keV})$ and magnetic fields have been interpreted as the result of large-scale flapping motions of Saturn's plasma and current sheet. A simple geometrical model of this flapping was constructed and fitted to electron densities measured in Saturn's magnetotail. In constructing such a model the location of the spacecraft with respect to the flapping plasma/current sheet is included and systematic errors in the inferred prime meridian longitudes are reduced.

[48] This model was fitted to electron number densities, measured by the CAPS/ELS instrument, for 10 passes of Cassini in the magnetotail during 2006. Overall, the model reasonably represents the periodic behavior of electron number densities and magnetic fields in Saturn's post-duskpost-midnight outer magnetosphere. Figure 10 illustrates the fitted plasma sheet parameters for each rev and complements the results in Table 2 . Solid symbols indicate outbound passes and open symbols inbound passes. The hinging distance and wave speed display significant scatter that is generally uncorrelated with the orbit of the spacecraft. The prime meridian of the model plasma sheet is somewhat more stable, and is well-ordered by the magnetic phase presented by Provan et al. [2009a] over more than eight months of in situ observations. This phase model was constructed from near equatorial magnetic field oscillations that have been shown to generally be in phase with southern SKR. The exception to this ordering is from the outbound passes of revs 25 and 26 in the post-dusk sector. We argued that a significant shift in the plasma sheet phase on the outbound pass of rev 26 was due to the influence of a different period signal originating in the northern hemisphere auroral zone. This effect is discussed in more detail by Provan et al. (submitted manuscript, 2011).

[49] The fitted plasma sheet hinging distances were found to range between 16 and $32 \mathrm{R}_{\mathrm{S}}$ for the 10 passes studied here. Variations in solar wind dynamic pressure have been shown to affect the hinging distance of Earth's magnetotail [e.g., Tsyganenko and Fairfield, 2004]. This is interpreted in terms in terms of pressure equilibrium in the tail. During increases in solar wind dynamic pressure, the magnetotail is compressed more or less symmetrically. With the plasma sheet nominally displaced north of the rotational equator during northern hemisphere winter, an imbalance in lobe magnetic pressure is produced. This imbalance results in pressure on the plasma sheet and moves the sheet north of the rotational equator to equalize the lobe magnetic pressure. Thus when the tail is compressed the plasma sheet moves northward (due to the decreased hinging distance) and may move sufficiently far to avoid detection by a spacecraft. Abrupt disappearances of the periodicity and simultaneous increases in tail lobe field are evidence of such a process and have also been discussed by Arridge et al. [2008b], Jackman et al. [2010], and André et al. (manuscript in preparation, 2011). Evidence for rapid variations in the hinging distance can also be identified in the data. The magnetic field data in Figure 3 indicate that Cassini encounters and sometimes crosses the magnetodisc current sheet, notably at 18 and $66 \mathrm{~h}$. It is clear from both the magnetometer data and the modeled spacecraft trajectory (in red in Figure 3c) that Cassini periodically gets very close to the center of the current sheet and occasionally crosses the center plane. Small, timedependent changes in the hinging distance can then either 

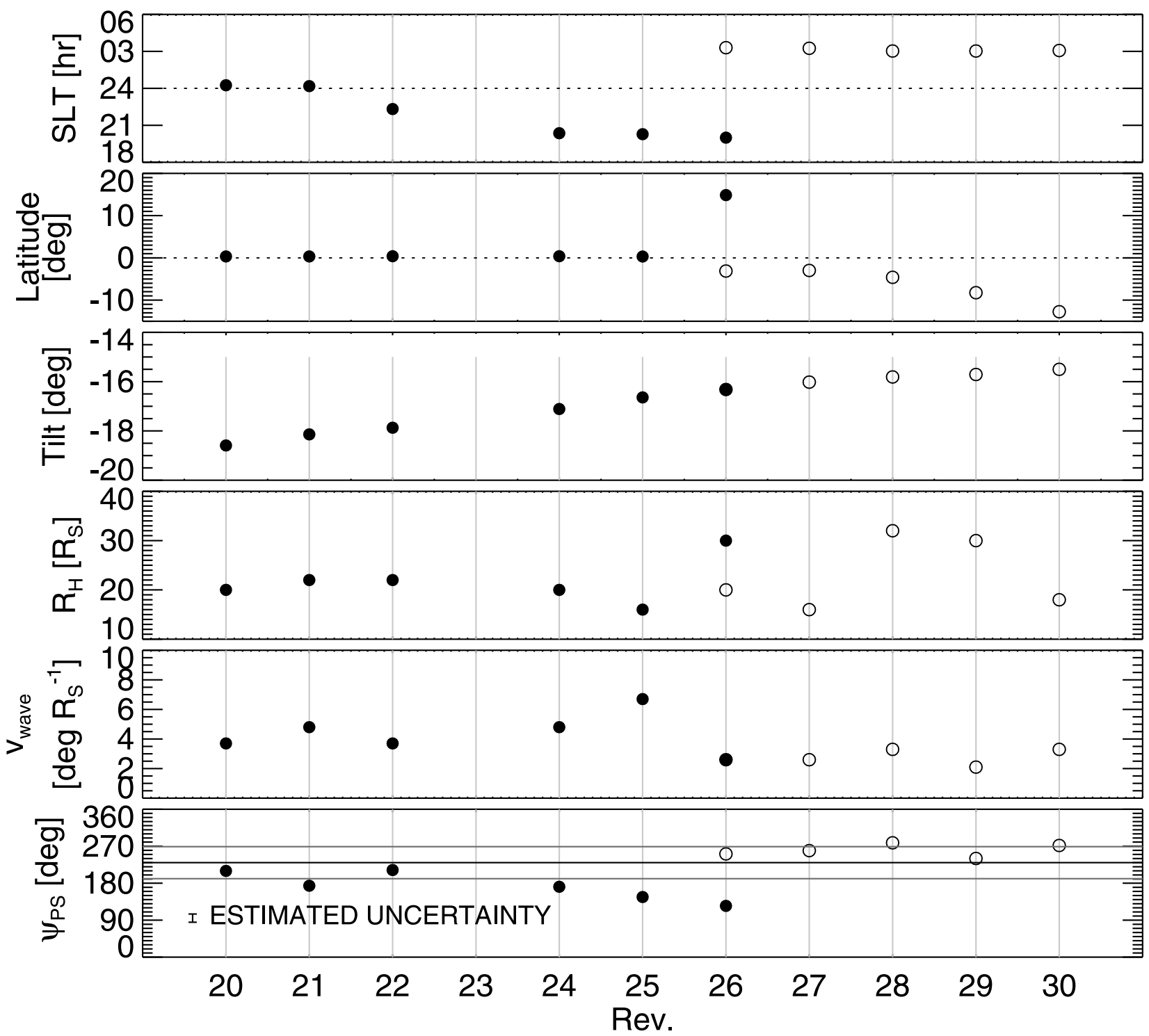

Figure 10. Summary of the modeling results obtained using the simple structural plasma sheet model. The local time and latitude of the spacecraft at $15 \mathrm{R}_{\mathrm{S}}$, the magnetospheric tilt angle $\theta_{\text {SUN }}$ (between Saturn's spin axis and the solar wind flow), and the fitted hinging distance, wave delay, and prime meridian are shown. Solid symbols show outbound passes, and open symbols show inbound passes. For the prime meridian, $\pm 20^{\circ}$ vertical error bars have been included as a guide to the (empirical) uncertainty. The horizontal solid lines for the prime meridian show the mean (black) of the modeled plasma sheet phases from passes in the midnight/post-midnight sector and one standard deviation from this mean (gray).

move the plasma sheet to produce a current sheet crossing, or move it away to produce a current sheet encounter.

[50] Modeled wave speeds were found to range between 5 and $16 \mathrm{R}_{\mathrm{S}} \mathrm{h}^{-1}\left(84-270 \mathrm{~km} \mathrm{~s}^{-1}\right)$, which is a factor of up to 5 larger than the $\sim 50 \mathrm{~km} \mathrm{~s}^{-1}$ fast mode speed presented by Cowley et al. [2006] but are in reasonable agreement with values for the Alfvén wave and sound speeds that can be made using Cassini plasma data. These plasma sheet wave speeds correspond to phase delays of between 2.1 and $6.7^{\circ} \mathrm{R}_{\mathrm{S}}^{-1}$ that are in approximate agreement with results presented by Carbary et al. [2007b] and Andrews et al. [2010a].

\subsection{Phase Relationships and Phase Shifts}

[51] Figure 11 illustrates the phase relationship between the magnetic oscillations in the core and the phase of vertical motion of the tail plasma sheet. The phases have all been plotted at a common radial distance of $12 R_{S}$ in order to deduce the relationships between the core and the flapping of the plasma sheet. The plasma sheet phase was evaluated at this radial distance and the magnetic core phase was evaluated at $12 \mathrm{R}_{\mathrm{S}}$ using the results of Andrews et al. [2010a].

[52] As a reminder of the nature of these phases, the $B_{r}$, $\mathrm{B}_{\theta}$, and $\mathrm{B}_{\varphi}$ components of the core field near local midnight have magnetic phases $\psi_{\mathrm{Br}}, \psi_{\mathrm{B} \theta}$, and $\psi_{\mathrm{B} \varphi}$ around 19,11 , and 


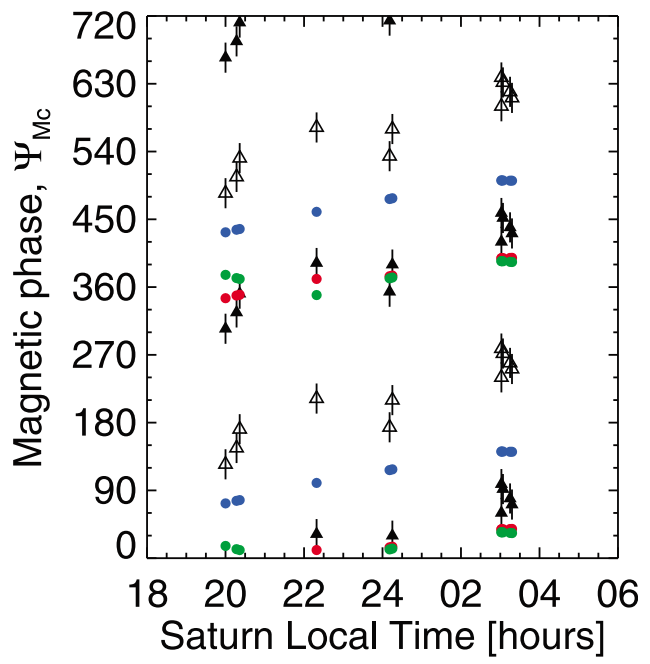

Figure 11. Phase relationships between the delayed core magnetic field perturbations [Andrews et al., 2010a] and the determined plasma sheet phase at a common radial distance of $12 \mathrm{R}_{\mathrm{S}}$. The red, green, and blue circles indicate the phase of the radial, polar, and azimuthal core magnetic field. Triangles indicate the fitted plasma sheet oscillation phase, with the open symbols indicating the fitted phase, and solid symbols indicating $\psi+180^{\circ}$ for ease of comparison with the magnetic oscillations. The vertical lines indicate the uncertainty on the plasma sheet phase. The phase relationships suggest that the plasma sheet oscillations are in anti-phase with the core radial field component in the midnight sector. In the pre-midnight sector the plasma sheet slightly leads in anti-phase and in the post-midnight sector it slightly lags this anti-phase relationship.

$110^{\circ}$, respectively. In the core region inside of $12 R_{S}$, the $\psi_{\mathrm{Br}}$ and $\psi_{\mathrm{B} \theta}$ phases are nominally zero and the $\psi_{\mathrm{B} \varphi}$ phase is nominally $90^{\circ}$ [Andrews et al., 2008]. When the subspacecraft magnetic phase $\Psi_{\mathrm{Mc}}$ equals $\psi_{\mathrm{Br}}$, the core field points radially outward; at $\psi_{\mathrm{B} \theta}$, the ring current is the weakest. This latter point is due to the fact that at this phase the $\mathrm{B}_{\theta}$ perturbation field is the weakest corresponding to the weakest azimuthal currents circulating in the core region. The plasma sheet phase $\psi_{\mathrm{PS}}$ is the phase at which the plasma sheet is at one of its vertical extrema and is defined as being at its largest displacement in the direction north of the rotational equator when $\Psi_{\mathrm{Mc}}=\psi_{\mathrm{PS}}$, and at its largest displacement south of the rotational equator at $\psi_{\mathrm{PS}}+180^{\circ}$. At a given radial distance the displacement at a phase $\psi_{\mathrm{PS}}+$ $180^{\circ}$ may result in a slight displacement of the plasma sheet south of the rotational equator or simply represent the closest approach of the plasma sheet to the rotational equator, depending on the extent of DC hinging of the plasma sheet.

[53] In describing the phase relationships we will refer to the phase $\psi_{\mathrm{PS}}$, where the plasma sheet is furthest from the equator. The open triangles in Figure 11 represent the plasma sheet phases listed in Table 2 (maximum $\left|z_{C S}\right|$ extremum) and the solid triangles indicate the largest displacement in a direction south of the equator (again, not necessarily crossing south of the rotational equator). The phase at which the core magnetic oscillations maximize (at positive values) is denoted by the colored filled circles: red
$\left(\psi_{\mathrm{Br}}\right)$, green $\left(\psi_{\mathrm{B} \theta}\right)$, and blue $\left(\psi_{\mathrm{B} \varphi}\right)$. The phases vary with local time according to the phases determined by Andrews et al. [2010a].

[54] Concentrating on the passes at midnight and just premidnight, one can see that the azimuthal core field (blue) is in lagging quadrature with the radial (red) and polar (green) components of the field. The plasma sheet is in anti-phase with the radial and azimuthal core field such that the sheet is closest to the equator (filled triangles) when the core radial field points outward and the ring current is weakest. Assuming that the plasma density asymmetry identified by Gurnett et al. [2007] maintains its phase relationship with the azimuthal core field, then the plasma sheet extrema are also in lagging quadrature with the plasma density asymmetry.

[55] For passes in the post-midnight sector, the plasma sheet phases lag those in the midnight sector by around $70^{\circ}$. The core field is similarly phase delayed, but not to such a large extent, although the uncertainties in the phases (of both the magnetic field in the core and the plasma sheet) may make this a weaker result than is first apparent. In the dusk/pre-midnight sector the plasma sheet phases lead those at midnight by around $40^{\circ}$. There is a similar lead in the core field but once again the delay is not to such a large extent as the plasma sheet.

[56] The phase of the plasma sheet motion in the postmidnight sector lags that in the pre-midnight sector by approximately $90^{\circ}$. These results and the case study analysis in section 4 show that while there is evidence for some jitter and unsteadiness in the phase of the plasma sheet oscillations, they are ordered by magnetic phase over more than eight months. There is little evidence of the persistent clock reset, which was postulated as one of the possible reasons for the spiral shifts observed by Carbary et al. [2007b]. We have also accounted for the geometrical effects discussed by Carbary et al. [2007b] and in section 1. The results are consistent with local time asymmetries in the phase of the global magnetospheric periodicities, but where the local time effects on the plasma sheet oscillations appears to be stronger than the effects on the core field. However, generally the results show that the flapping of the plasma sheet is in anti-phase with $B_{r}$ to a reasonable approximation.

[57] Figure 12 schematically shows the relationship between the core field and the motion of the magnetotail. Figure 12a shows the geometry of the plasma sheet relative to the equator in a noon-midnight meridional slice (not to scale, especially on the dayside) at several values of the plasma sheet phase $\Psi_{\mathrm{PS}}$. Figure 12b (following Clarke et al. [2010]) shows the relationship between the density asymmetry in the inner magnetosphere [Gurnett et al., 2007] (the filled segments close to the planet), the core magnetic field [Provan et al., 2009a], and the identified plasma sheet flapping extrema (red and blue curves). This is drawn for the phase where the (southern) SKR intensity is a maximum. This pattern then rotates anti-clockwise (prograde) at the southern SKR period. One can see that the extrema are in phase with core $B_{r}$ extrema such that the displacements of the plasma sheet north of the rotational equator are in phase with inwardly oriented core magnetic fields. The phase fronts of the plasma sheet flapping are delayed, forming spirals with a wavelength of (peak to trough) of around $40 R_{S}$. At this particular subsolar phase the magnetopause is displaced outwards in the post-noon sector [Clarke et al., 2010], 

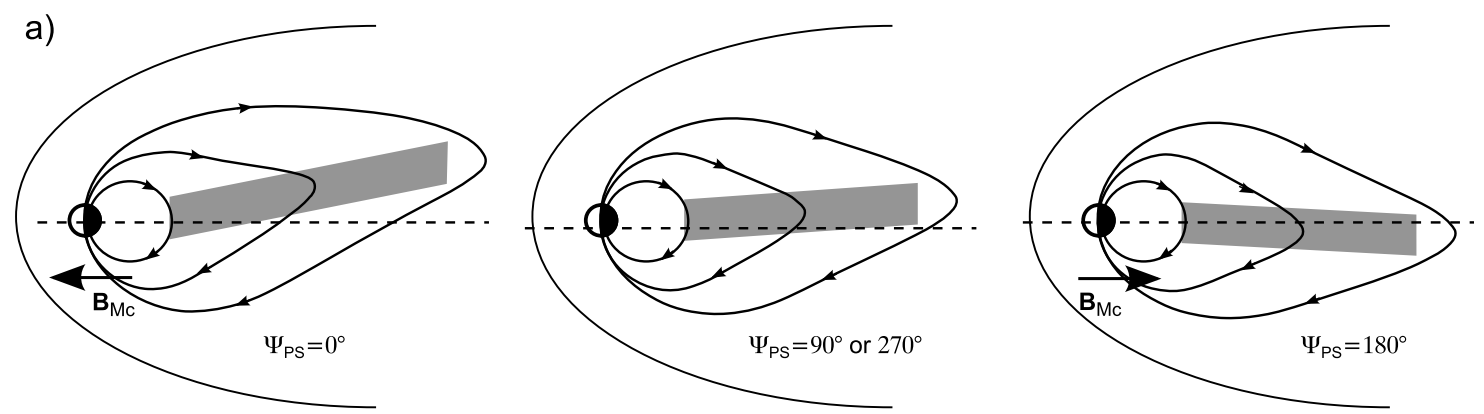

b)

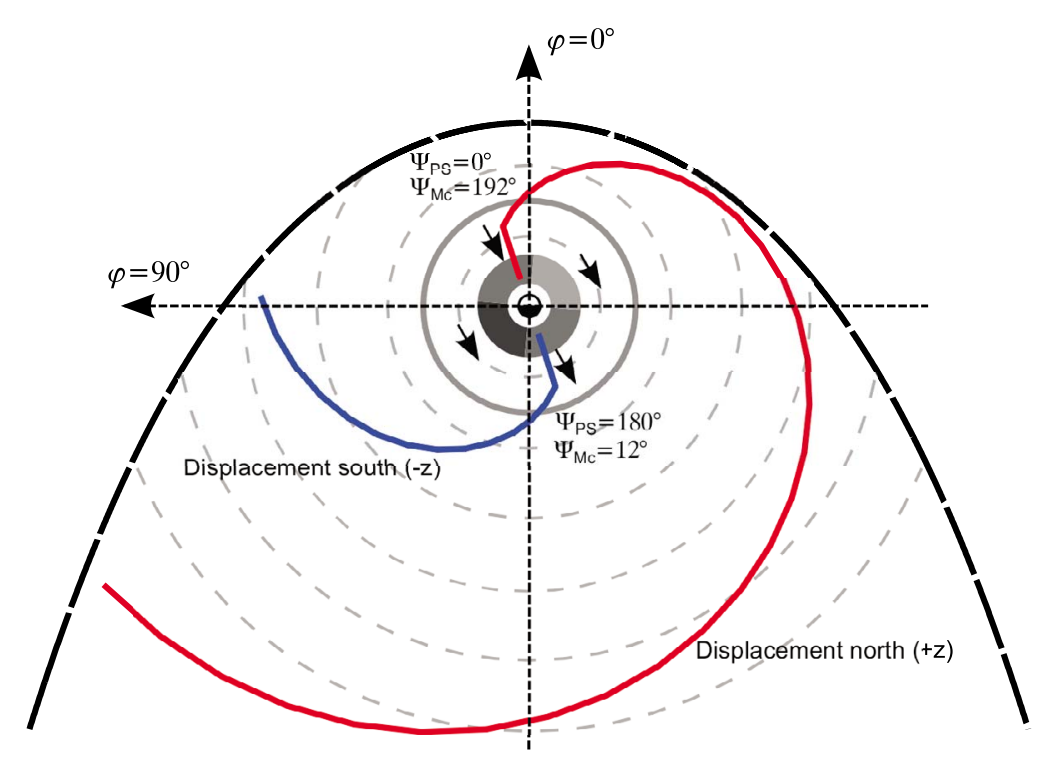

Figure 12. Schematic illustration showing the identified phase relationships. (a) The inferred plasma sheet and magnetic field configuration at various phases of the flapping motion from its extreme displacement away from the equator, to a nominally hinged configuration, to being slightly displaced below the equator. (b) and the core magnetic field, inner magnetospheric density asymmetry, and plasma sheet extrema [after Clarke et al., 2010]. The diagram is drawn for a subsolar magnetic phase of $210^{\circ}$ corresponding to maximum intensity of SKR (south). At this time the core magnetic field points radially outward at around $0200 \mathrm{~h}$ Saturn local time and inward around 1400. The minimum (actually slightly below the equator) $\mathrm{AC}$ displacement lies along the blue curve and forms a swept-back spiral produced by radial delays. The maximum displacement above the equator is along the red curve. The density asymmetry in the inner magnetosphere has a maximum density near $\Psi_{\mathrm{Mc}}=80^{\circ}$ (depicted by the dark segment) and is in the post-dusk sector. The magnetopause is indicated by the black curve, and the solid gray curve indicates the outer boundary of the cam region at $15 R_{S}$.

approximately lining up with the plasma sheet adopting a nominally warped (where the "AC" plasma sheet oscillation term is zero and the plasma sheet is in the nominally warped position) configuration between the two vertical extrema.

\subsection{Implications for Models of Magnetospheric Periodicities}

[58] A number of conceptual and theoretical models have been developed to explain the large-scale periodicities in Saturn's magnetotail. Carbary et al. [2007a] postulated that a compressive motion in the inner magnetosphere would periodically push out the tail when high-pressure sector was located on the nightside. For an observer located inside the plasma sheet in the low pressure sector, the plasma sheet would then move northward of the observer in the high pressure sector. Hence the plasma sheet would move up in the high pressure sector, and down in the low pressure sector. Clarke et al. [2010] have shown that the high pressure sector identified by Burch et al. [2009] and the density asymmetry reported by Gurnett et al. [2007] are in phase with an azimuthal orientation of the core magnetic field and outward displacements of the magnetopause. This shows that high pressure sectors in the magnetosphere are in quadrature with flapping motions in the outer magnetosphere. Thus, these results do not appear to support the model proposed by Carbary et al. [2007a], although further work mapping high pressure sectors in the magnetosphere and the evolution of their phase with radial distance and local time would be beneficial.

[59] Southwood and Kivelson [2007] have argued that the cam field in the inner magnetosphere generates an effective dipole tilt in the outer magnetosphere, with a magnitude of 
$12^{\circ}-15^{\circ}$. When $\Delta \mathrm{B}_{\mathrm{r}}$ reaches a positive maximum in the middle magnetosphere near $\sim 15 \mathrm{R}_{\mathrm{S}}$ the current sheet moves down (south), and when $\Delta \mathrm{B}_{\mathrm{r}}$ reaches a negative maximum the plasma sheet moves up (north). Hence the plasma sheet extrema in the outer magnetosphere should be in phase with $\Delta \mathrm{B}_{\mathrm{r}}$ extrema in the middle magnetosphere. The phase relationship between the plasma sheet and the radial core magnetic field perturbation supports this model. This tilting behavior and phase relationship with the core field is also consistent with the model presented by Provan et al. [2009a, Figure 11b], which is essentially an elaboration on the ideas of Southwood and Kivelson [2007], including an explanation of the polar (co-latitudinal) magnetic field perturbations, and the model of Andrews et al. [2010b], which extends these to include separate north and south systems.

[60] Finally, Khurana et al. [2009] have proposed that a longitudinally asymmetric lobe field produced by a partial ring current, rotating at the magnetospheric period, interacts with the solar wind to asymmetrically lift the outer magnetosphere. This produces an effective modulation of the plasma sheet hinging distance with time for a stationary observer. When the plasma sheet hinging distance is large (small) the plasma sheet is close to (far from) the equator. Khurana et al. [2009] showed that when the ring current maximized in strength, the $\mathrm{B}_{\theta}$ perturbation field maximized and thus the lobe field at that phase was increased due to the expulsion of magnetic flux from the near-equatorial plasma sheet. Enhanced magnetic pressure in the lobes more easily stands off the solar wind and thus the plasma sheet lies closer to the equator. This allows one to understand the phase relationships between the core field and the flapping of the plasma sheet in this model. A larger $\mathrm{B}_{\theta}$ core perturbation field (smaller total field) will result in the plasma sheet moving toward the equator. The plasma sheet phase in the present study (the magnetic phase at which the plasma sheet is farthest from the equator) should be in phase with a weaker ring current, a smaller $\mathrm{B}_{\theta}$ core perturbation field, and a larger total $\mathrm{B}_{\theta}$ in the core. From the results in Figures 11 and 12 one can see that negative plasma sheet extrema are approximately in phase with the $\mathrm{B}_{\mathrm{r}}$ and $\mathrm{B}_{\theta}$ perturbation fields such that when the plasma sheet is at a southerly extrema (furthest downward extent), the core $\mathrm{B}_{\mathrm{r}}$ and $\mathrm{B}_{\theta}$ are positive and thus in a state where the ring current is weakest. This is apparently opposite (by $180^{\circ}$ ) to the predictions of the Khurana et al. [2009] model. However, caution must be applied to this conclusion since the flapping oscillations in this model are controlled by the interaction between the lobe field and the solar wind. Khurana et al. [2009] clearly show that the lobe field is modulated periodically but the phase delays in this lobe field modulation have yet to be established. The use of near equatorial phase relationships may not be valid in testing this model against the phase of the plasma sheet flapping. However, this does provide information about the phase relationship between the flapping of the plasma sheet and a rotating partial ring current that may be important in developing future models or refining existing ones.

[61] As pointed out by Khurana et al. [2009], a powerful tool for understanding the flapping of the plasma sheet are polarity maps for the sign of the $\mathrm{B}_{\mathrm{r}}$ component of the (total) magnetic field [see Khurana et al., 2009, Figure 8]. These maps show that the $B_{r}$ component only reverses sign near the equator and at larger (positive) latitudes, which is a natural prediction of their asymmetric lift model. Figure 13 shows a similar polarity map using the model equations presented in section 3. One can see that in general the model polarity map is in good agreement with that presented by Khurana et al. [2009], in that there is an extended region at positive latitudes and a region around the equator where the $\mathrm{B}_{\mathrm{r}}$ component can reverse in sign. This supports the model of Khurana et al. [2009] but also shows that a conceptual model where the magnetosphere develops an effective dipole tilt [e.g., Southwood and Kivelson, 2007; Provan et al., 2009a] beyond a certain distance can produce a polarity map that mimics the asymmetric lift model. This clearly shows that more work is required to understand the relationship between motions in the outer magnetosphere and other periodic phenomena in the magnetosphere, particularly relating to distinct magnetospheric periods in the northern and southern auroral regions.

\subsection{Plasma Sheet Speed and Acceleration}

[62] The model described by equations (4) and (5) gives the time-dependent position of the plasma and so can be differentiated to give the vertical speed $\mathrm{u}_{\mathrm{z}}$ and acceleration $\partial \mathrm{u}_{\mathrm{z}} / \partial \mathrm{t}$ of the plasma sheet. Equation (8) presents these derivatives, where the DC hinging term is constant and becomes zero and terms involving the derivative of the period (second derivative of the magnetic phase) have been set to zero.

$$
\begin{aligned}
u_{z}(t, \varphi, \rho) & =-\left(\rho-\rho_{0}\right) \Omega \tan \theta_{T I L T} \sin \Psi_{P S}(t, \varphi, \rho) \\
\frac{\partial u_{z}(t, \varphi, \rho)}{\partial t} & =-\left(\rho-\rho_{0}\right) \Omega^{2} \tan \theta_{T I L T} \cos \Psi_{P S}(t, \varphi, \rho) .
\end{aligned}
$$

The maximum speed of this current sheet is obtained when $\Psi_{\mathrm{PS}}=90^{\circ}$ and maximum acceleration when $\Psi_{\mathrm{PS}}=0^{\circ}$. Using values of $\rho_{0}=12 \mathrm{R}_{\mathrm{S}}$ and $\theta_{\mathrm{TILT}}=12^{\circ}$, one finds values of $\mathrm{u}_{\mathrm{z}}\left(\Psi_{\mathrm{PS}}=90^{\circ}\right)=17 \mathrm{~km} \mathrm{~s}^{-1}$ and $\partial \mathrm{u}_{\mathrm{z}} / \partial \mathrm{t}\left(\Psi_{\mathrm{PS}}=0^{\circ}\right)=29 \mathrm{~m} \mathrm{~s}^{-2}$ at Titan's orbital distance. These plasma sheet velocities and accelerations may provide additional useful constraints for understanding periodicities in Saturn's magnetosphere and the magnetic stresses associated with periodically moving the plasma sheet.

\subsection{Future Work}

[63] Krupp et al. [2005] showed that the energetic particle fluxes and magnetic field strength exhibited an in-phase modulation during the outbound pass of rev B at moderately high latitudes. Such compressional behavior in the lobes must undergo a transition to the transverse flapping motion of the tail. Khurana et al. [2009] have emphasized the importance of the compressional lobe modulations by suggesting that they generate the transverse motion of the tail. A priori, there is no reason to expect that the transverse current/plasma sheet motion and lobe compressional mode are in phase and hence, there may be some interaction between the two modes, perhaps involving a sausage mode plasma sheet behavior driven by the compressional lobe modulation as suggested by Morooka et al. [2009]. At high latitudes far from the plasma sheet, compressional fluctuations are observed in the lobes. Closer to the plasma sheet the transverse oscillation of the plasma sheet must be modulated by the sausage mode oscillation of the plasma sheet. Finally, near the center of the plasma sheet oscillations, the dominant 


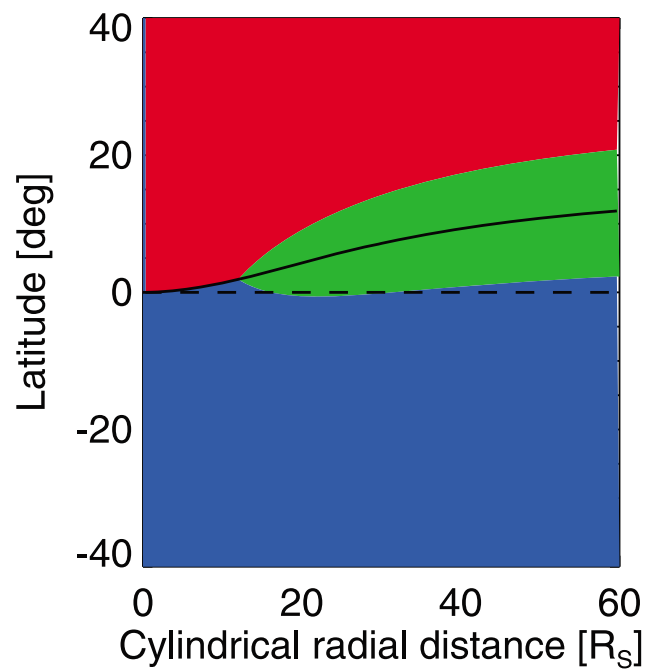

Figure 13. Schematic diagram, using the model equations developed in section 3 , showing the regions where the radial magnetic field component would be found to be positive (red), negative (blue), and reversing in sign (green) and is directly comparable to Figures 7 and 8 of Khurana et al. [2009]. The mean location of the current sheet, according to the model of Arridge et al. [2008b] is illustrated by the solid line through the green region, and the dashed line represents the Kronographic equator. This diagram has been drawn for a hinging distance of $20 \mathrm{R}_{\mathrm{S}}$, a current sheet tilt angle of $12^{\circ}$, and a magnetospheric tilt angle of $17.5^{\circ}$.

modulation will be caused by the transverse motion of the sheet. The identification of distinct northern and southern auroral magnetospheric periods and potentially distinct northern and southern lobe compressional periods would considerably complicate this picture (Provan et al., submitted manuscript, 2011). The expected features of such a scenario have yet to be examined in detail and are worthy of future study. The unambiguous detection of transverse and compressional plasma sheet behavior would represent an important clue regarding periodicities in Saturn's magnetosphere.

[64] Finally, in the modeling reported here, fixed values for the hinging distance and wave velocity were adopted for each pass. In reality these parameters are also a function of local time (Andrews et al. [2010a] have found such wave speed asymmetries), and perhaps also radial distance in the case of the wave velocity. Future work should attempt to take this into account when a more complete understanding of the hinging of Saturn's plasma sheet is available.

\section{Summary}

[65] In this paper the motion of Saturn's magnetotail plasma sheet has been investigated using a simple structural model. Phase relationships have been determined between the motion of the plasma sheet, established magnetic field periodicities in the inner magnetosphere, and SKR (via the proxy of SLS3/magnetic phase). It is clear that whichever internal mechanism produces periodicities in SKR and magnetic fields in the cam/core region, is also strongly related to the production of global plasma sheet flapping motions, albeit preferentially controlled by current systems in the southern hemisphere. The prime meridian of the plasma sheet oscillations was found to be relatively stable with a strong local time dependence that is not fully accounted for by local time variability in the core magnetic field [Andrews et al., 2010a]. However, to a first approximation the plasma sheet extrema were in phase with the radial component of the core magnetic field such that the radial field points outward when the plasma sheet is at its most southerly extrema.

[66] Shifts in the phase of the plasma sheet oscillations do not support the idea of a "clock reset" but can be attributed to local time asymmetries in the magnetosphere and possibly beating between the northern and southern SKR periods. The established phase relationships support the effective tilted dipole model advanced by Southwood and Kivelson [2007] and expanded on by Provan et al. [2009a] and Andrews et al. [2010b], but present some inconsistencies with the asymmetric lift model of Khurana et al. [2009] that may be resolved by a study of the phase behavior of the lobe field and the pressure-driven model of Carbary et al. [2007a]. Carbary et al. [2008] used ENA images to demonstrate a periodic tilting of Saturn's plasma sheet that had a phase of $47^{\circ}$ in SLS3 longitude, which corresponds to approximately $300^{\circ}$ in magnetic phase, which is in approximate quadrature with the plasma sheet phases identified in the midnight local time sector in this study. However, different radial distance ranges between this study and Carbary et al. [2008] imply that some understanding of phase propagation is required to fully understand the relationship between the results of Carbary et al. [2008] and the results presented here.

[67] We have demonstrated robust phase relationships between flapping the plasma sheet in the nightside outer magnetosphere and the near-equatorial magnetic field oscillations in the core quasi-dipolar region of the magnetosphere inside $12 \mathrm{R}_{\mathrm{S}}$. These relationships will be useful in testing and refining models of periodicities in Saturn's magnetosphere.

\section{Appendix A: Density Model}

[68] To fit for the plasma sheet parameters, it was found necessary to develop a simple model to represent the central plasma sheet density as a function of radial distance. Survey results presented by Arridge et al. [2009] were used to extract electron moments close to the center of the plasma sheet. As discussed by Arridge et al. [2009], large values of partial $(0.5 \mathrm{eV}<\mathrm{E}<28 \mathrm{keV})$ electron beta are necessary but not sufficient to uniquely identify the center of the plasma sheet. To properly extract central plasma sheet samples, three criteria were applied electron moments: $\beta_{\mathrm{e}}>0.01, \mathrm{~T}_{\mathrm{e}}<$ $400 \mathrm{eV}$ (to remove energetic disturbed states of the plasma sheet), and field stretch angles less than $30^{\circ}$ (essentially the angle the field line makes with the equatorial plane, defined as $\left.\tan ^{-1}\left(\mathrm{~B}_{\mathrm{r}}^{2}+\mathrm{B}_{\varphi}^{2}\right)^{1 / 2} / \mathrm{B}_{\theta}\right)$.

[69] Using these criteria, 3871 central plasma sheet samples were extracted and fitted using linear least squares (in $\log -\log$ space) to a power law: $n_{0}(r)=a^{b}$, where uncertainties on the density were accounted for in the fit. The best fit parameters were found to be $\mathrm{a}=5.62 \times 10^{6} \mathrm{~m}^{-3}$ and $\mathrm{b}=$ -1.67 . Figure A1 illustrates the points used in the fit and the best fit power law. To test the effect of varying the data 


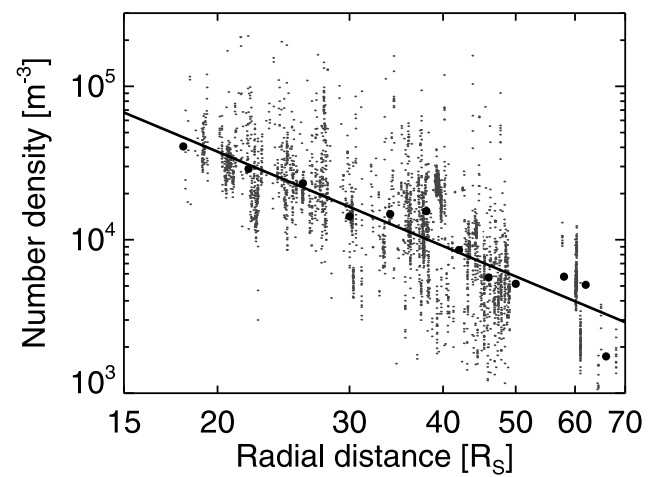

Figure A1. Electron number density in the central plasma sheet as a function of radial distance from Saturn with a best fit power law $\mathrm{n}_{\mathrm{e}}=\left(5.62 \times 10^{6}\right) \mathrm{r}^{-1.67}$.

selection criteria on the stability of these parameters the cutoff electron temperature and stretch angles were varied and sets of fit parameters obtained. For stretch angles of 30 , 40,50 , and $60^{\circ}$, partial electron betas of $0.005,0.01$, and 0.05 , and temperatures 400 and $600 \mathrm{eV}$ the power law exponent, $\mathrm{b}$ was found to vary between -1.64 and -2.10 and the constant, a between $4.92 \times 10^{6} \mathrm{~m}^{-3}$ and $2.87 \times 10^{7} \mathrm{~m}^{-3}$.

\section{Appendix B: Model Expressed in SLS3 Longitude}

[70] The plasma sheet model expressed as a function of SLS3 longitude, $\lambda_{\mathrm{SLS} 3}$, is

$$
z_{C S}(\rho)=\left[\rho-r_{H} \tanh \left(\frac{\rho}{r_{H}}\right)\right] \tan \theta_{S U N}+\left(\rho-\rho_{0}\right) \tan \theta_{T I L T} \cos \lambda,
$$

where $\lambda$ is the phase of oscillation of the plasma sheet, analogous to $\Psi_{\mathrm{PS}}$ in equation (5) and is given by

$$
\lambda=\lambda_{S L S 3}-\lambda_{0}-\frac{\Omega_{S K R}\left(\rho-\rho_{0}\right)}{v_{W A V E}} .
$$

Here $\lambda_{0}$ is the prime meridian, similar to $\psi_{\mathrm{PS}}$, but which includes the $100^{\circ}$ offset between the SLS3 and the SKR phase, and the drift in phase between SLS3 and the magnetic core phase reported by Provan et al. [2009a].

\section{Appendix C: Justification for Harris Density Profile}

[71] The magnetic field pressure associated with a Harris sheet is proportional to $\tanh ^{2}\left(z^{\prime} / H\right)$, where $z^{\prime}$ is the distance from the center of the plasma sheet along the local normal to the sheet and $\mathrm{H}$ is the scale height of the sheet, and assuming tangential pressure balance it can be shown that the plasma pressure in the sheet is $\mathrm{p}(\mathrm{z})=\mathrm{p}_{0} \operatorname{sech}^{2}\left(\mathrm{z}^{\prime} / \mathrm{H}\right)$, where $p_{0}$ is the plasma pressure at the center of the sheet. Using the ideal gas equation one can hence write that $\mathrm{n}(\mathrm{z}) \mathrm{T}(\mathrm{z})=\mathrm{n}_{0} \mathrm{~T}_{0} \operatorname{sech}^{2}\left(\mathrm{z}^{\prime} / \mathrm{H}\right)$. The observations presented in Figure 2 show that to a reasonable approximation the plasma sheet temperature $\mathrm{T}$ is independent of $\mathrm{z}^{\prime}$ [see also Arridge et al., 2009]. Not only does this allow us to eliminate $\mathrm{T}$ from both sides of this equation since $\mathrm{T}(\mathrm{z})=\mathrm{T}_{0}$ but also this simplifies the vertical profile as in reality $\mathrm{H}=\mathrm{H}(\mathrm{T})$ and a constant temperature this implies a constant scale height. Hence this allows us to write the density profile as

$$
n=n_{0} \operatorname{sech}^{2}\left(\frac{z^{\prime}}{H}\right)
$$

[72] Acknowledgments. We thank L. K. Gilbert and G. R. Lewis for data processing and software support for CAPS at MSSL, and S. Kellock, L. Nani-Alconcel, and P. Slootweg at Imperial College for MAG data processing. CSA thanks J. F. Carbary, D. E. Jones, M. G. Kivelson, D. G. Mitchell, and D. J. Southwood for useful and thought provoking discussions, and K. H. Arridge for useful comments on the manuscript. C.S.A. and A.J.C. were supported in this work by the STFC rolling grant to MSSL/UCL, C.S.A. was also supported by an STFC Postdoctoral fellowship and C.M.J. was also supported by the STFC rolling grant to Imperial College. C.T.R. and K.K.K. were supported by NASA. S.W.H. C. and G.P. were supported by the STFC rolling grant to RSPP/Leicester and D.J.A. by an STFC Quota Studentship. C.S.A., N.A., K.K.K., H.J. M., C.T.R., and E.C.S. acknowledge funding and support from the International Space Science Institute, where part of this work was discussed. Cassini CAPS and MAG operations activities at MSSL and Imperial College were supported by STFC.

[73] Masaki Fujimoto thanks the reviewers for their assistance in evaluating this paper.

\section{References}

Andrews, D. J., E. J. Bunce, S. W. H. Cowley, M. K. Dougherty, G. Provan, and D. J. Southwood (2008), Planetary period oscillations in Saturn's magnetosphere: Phase relation of equatorial magnetic field oscillations and Saturn kilometric radiation modulation, J. Geophys. Res., 113, A09205, doi:10.1029/2007JA012937.

Andrews, D. J., S. W. H. Cowley, M. K. Dougherty, and G. Provan (2010a), Magnetic field oscillations near the planetary period in Saturn's equatorial magnetosphere: Variation of amplitude and phase with radial distance and local time, J. Geophys. Res., 115, A04212, doi:10.1029/ 2009JA014729.

Andrews, D. J., A. J. Coates, S. W. H. Cowley, M. K. Dougherty, L. Lamy, G. Provan, and P. Zarka (2010b), Magnetospheric period oscillations at Saturn: Comparison of equatorial and high-latitude magnetic field periods with north and south Saturn kilometric radiation periods, J. Geophys. Res., 115, A12252, doi:10.1029/2010JA015666.

Arridge, C. S., N. André, N. Achilleos, K. K. Khurana, C. L. Bertucci, L. K. Gilbert, G. R. Lewis, A. J. Coates, and M. K. Dougherty (2008a), Thermal electron periodicities at $20 \mathrm{R}_{\mathrm{S}}$ in Saturn's magnetosphere, Geophys. Res. Lett., 35, L15107, doi:10.1029/2008GL034132.

Arridge, C. S., K. K. Khurana, C. T. Russell, D. J. Southwood, N. Achilleos, M. K. Dougherty, A. J. Coates, and H. K. Leinweber (2008b), Warping of Saturn's magnetospheric and magnetotail current sheets, J. Geophys. Res. 113, A08217, doi:10.1029/2007JA012963.

Arridge, C. S., et al. (2009), Plasma electrons in Saturn's magnetotail: Structure, distribution and energisation, Planet. Space Sci., 57(14-15), 2032-2047, doi:10.1016/j.pss.2009.09.007.

Brandt, P. C., K. K. Khurana, D. G. Mitchell, N. Sergis, K. Dialynas, J. F. Carbary, E. C. Roelof, C. P. Paranicas, S. M. Krimigis, and B. H. Mauk (2010), Saturn's periodic magnetic field perturbations caused by a rotating partial ring current, Geophys. Res. Lett., 37, L22103, doi:10.1029/ 2010GL045285.

Burch, J. L., J. Goldstein, P. Mokashi, W. S. Lewis, C. Paty, D. T. Young, A. J. Coates, M. K. Dougherty, and N. André (2008), On the cause of Saturn's plasma periodicity, Geophys. Res. Lett., 35, L14105, doi:10.1029/2008GL034951.

Burch, J. L., A. D. DeJong, J. Goldstein, and D. T. Young (2009), Periodicity in Saturn's magnetosphere: Plasma cam, Geophys. Res. Lett., 36, L14203, doi:10.1029/2009GL039043.

Carbary, J. F., and S. M. Krimigis (1982), Charged particle periodicity in the Saturnian magnetosphere, Geophys. Res. Lett., 9(9), 1073-1076, doi:10.1029/GL009i009p01073.

Carbary, J. F., D. G. Mitchell, S. M. Krimigis, D. C. Hamilton, and N. Krupp (2007a), Spin-period effects in magnetospheres with no axial tilt, Geophys. Res. Lett., 34, L18107, doi:10.1029/2007GL030483.

Carbary, J. F., D. G. Mitchell, S. M. Krimigis, and N. Krupp (2007b), Evidence for spiral pattern in Saturn's magnetosphere using the new 
SKR longitudes, Geophys. Res. Lett., 34, L13105, doi:10.1029/ 2007GL030167.

Carbary, J. F., D. G. Mitchell, P. Brandt, E. C. Roelof, and S. M. Krimigis (2008), Periodic tilting of Saturn's plasma sheet, Geophys. Res. Lett., 35 L24101, doi:10.1029/2008GL036339.

Clarke, K. E., D. J. Andrews, C. S. Arridge, A. J. Coates, and S. W. H. Cowley (2010), Magnetopause oscillations near the planetary period at Saturn: Occurrence, phase, and amplitude, J. Geophys. Res., 115, A08209, doi:10.1029/2009JA014745.

Cowley, S. W. H., D. M. Wright, E. J. Bunce, A. C. Carter, M. K. Dougherty, G. Giampieri, J. D. Nichols, and T. R. Robinson (2006), Cassini observations of planetary-period magnetic field oscillations in Saturn's magnetosphere: Doppler shifts and phase motion, Geophys. Res. Lett., 33, L07104, doi:10.1029/2005GL025522.

Desch, M. D., and M. L. Kaiser (1981), Voyager measurement of the rotation period of Saturn's magnetic field, Geophys. Res. Lett., 8(3), 253-256, doi:10.1029/GL008i003p00253.

Espinosa, S. A., D. J. Southwood, and M. K. Dougherty (2003a), Reanalysis of Saturn's magnetospheric field data view of spin-periodic perturbations, J. Geophys. Res., 108(A2), 1085, 8 pp., doi:10.1029/2001JA005083.

Espinosa, S. A., D. J. Southwood, and M. K. Dougherty (2003b), How can Saturn impose its rotation period in a noncorotating magnetosphere? J. Geophys. Res., 108(A2), 1086, 8 pp., doi:10.1029/2001JA005084.

Gurnett, D. A., et al. (2005), Radio and plasma wave observations at Saturn from Cassini's approach and first orbit, Science, 307, 1255-1259, doi:10.1126/science. 1105356

Gurnett, D. A., A. M. Persoon, W. S. Kurth, J. B. Groene, T. F. Averkamp M. K. Dougherty, and D. J. Southwood (2007), The variable rotation period of the inner region of Saturn's plasma disk, Science, 316, 442-445, doi:10.1126/science.1138562

Gurnett, D. A., A. Lecacheux, W. S. Kurth, A. M. Persoon, J. B. Groene L. Lamy, P. Zarka, and J. F. Carbary (2009), Discovery of a north-south asymmetry in Saturn's radio rotation period, Geophys. Res. Lett., 36 , L16102, doi:10.1029/2009GL039621.

Gurnett, D. A., J. B. Groene, A. M. Persoon, J. D. Menietti, S.-Y. Ye, W. S. Kurth, R. J. MacDowall, and A. Lecacheux (2010), The reversal of the rotational modulation rates of the north and south components of Saturn kilometric radiation near equinox, Geophys. Res. Lett., 37, L24101, doi:10.1029/2010GL045796.

Jackman, C. M., and C. S. Arridge (2011), Statistical properties of the magnetic field in the Kronian magnetotail lobes and current sheet, J. Geophys. Res., 116, A05224, 8 pp. doi:10.1029/2010JA015973.

Jackman, C. M., C. S. Arridge, H. J. McAndrews, M. G. Henderson, and R. J. Wilson (2009a), Northward field excursions in Saturn's magnetotail and their relationship to magnetospheric periodicities, Geophys. Res. Lett., 36, L16101, doi:10.1029/2009GL039149.

Jackman, C. M., L. Lamy, M. P. Freeman, P. Zarka, B. Cecconi, W. S Kurth, S. W. H. Cowley, and M. K. Dougherty (2009b), On the character and distribution of lower-frequency radio emissions at Saturn, and their relationship to substorm-like events, J. Geophys. Res., 114, A08211, doi: 10.1029/2008JA013997.

Jackman, C. M., C. S. Arridge, J. A. Slavin, S. E. Milan, L. Lamy, M. K. Dougherty, and A. J. Coates (2010), In situ observations of the effect of a solar wind compression on Saturn's magnetotail, J. Geophys. Res., 115 A10240, doi:10.1029/2010JA015312.

Kellett, S., E. J. Bunce, A. J. Coates, and S. W. H. Cowley (2009), Thickness of Saturn's ring current determined from north-south Cassini passes through the current layer, J. Geophys. Res., 114, A04209, doi:10.1029/ 2008JA013942.

Khurana, K. K., and H. K. Schwarzl (2005), Global structure of Jupiter's magnetospheric current sheet, J. Geophys. Res., 110, A07227, 12 pp., doi:10.1029/2004JA010757.

Khurana, K. K., D. G. Mitchell, C. S. Arridge, M. K. Dougherty, C. T. Russell, C. Paranicas, N. Krupp, and A. J. Coates (2009), Sources of rotational signals in Saturn's magnetosphere, J. Geophys. Res., 114, A02211, 11 pp., doi:10.1029/2008JA013312.

Kivelson, M. G., P. J. Coleman, L. Froidevaux, and R. L. Rosenberg (1978), A time dependent model of the jovian current sheet, J. Geophys. Res., 83(A10), 4823-4829, doi:10.1029/JA083iA10p04823.

Krupp, N., et al. (2005), The saturnian plasma sheet as revealed by energetic particle measurements, Geophys. Res. Lett., 32, L20S03, 5 pp., doi:10.1029/2005GL022829.
Kurth, W. S., T. F. Averkamp, D. A. Gurnett, J. B. Groene, and A. Lecacheux (2008), An update to a Saturnian longitude system based on kilometric radio emissions, J. Geophys. Res., 113, A05222, 10 pp., doi:10.1029/ 2007JA012861

Lamy, L. (2011), Variability of Southern and Northern SKR Periodicities, Proc. 7th International Workshop on Planetary, Solar and Heliospheric Radio Emissions, in press.

Morooka, M. W., et al. (2009), The electron density of Saturn's magnetosphere, Ann. Geophys., 27, 2971-2991, doi:10.5194/angeo-27-2971-2009.

Nichols, J. D., J. T. Clarke, S. W. H. Cowley, J. Duval, A. J. Farmer, J.-C. Gérard, D. Grodent, and S. Wannawichian (2008), Oscillation of Saturn's southern auroral oval, J. Geophys. Res., 113, A11205, 12 pp., doi:10.1029/ 2008JA013444.

Press, W. H., S. A. Teukolsky, W. T. Vetterling, and B. P. Flannery (1992), Numerical Recipes in C: The Art of Scientific Computing, 2nd ed., Cambridge Univ. Press, Cambridge.

Provan, G., D. J. Andrews, C. S. Arridge, A. J. Coates, S. W. H. Cowley, S. E. Milan, M. K. Dougherty, and D. M. Wright (2009a), Polarization and phase of planetary-period magnetic field oscillations on high-latitude field lines in Saturn's magnetosphere, J. Geophys. Res., 114, A02225, doi:10.1029/2008JA013782.

Provan, G., S. W. H. Cowley, and J. D. Nichols (2009b), Phase relation of oscillations near the planetary period of Saturn's auroral oval and the equatorial magnetospheric magnetic field, J. Geophys. Res., 114 A04205, 15 pp., doi:10.1029/2008JA013988.

Provan, G., D. J. Andrews, B. Cecconi, S. W. H. Cowley, M. K. Dougherty, L. Lamy, and P. M. Zarka (2011), Magnetospheric period magnetic field oscillations at Saturn: Equatorial phase "jitter" produced by superposition of southern and northern period oscillations, J. Geophys. Res., 116 , A04225, 22 pp., doi:10.1029/2010JA016213.

Southwood, D. J., and M. G. Kivelson (2007), Saturnian magnetospheric dynamics: Elucidation of a camshaft model, J. Geophys. Res., 112, A12222, doi:10.1029/2007JA012254

Thomsen, M. F., D. B. Reisenfeld, D. M. Delapp, R. L. Tokar, D. T. Young, F. J. Crary, E. C. Sittler, M. A. McGraw, and J. D. Williams (2010), Survey of ion plasma parameters in Saturn's magnetosphere, J. Geophys. Res., 115, A10220, doi:10.1029/2010JA015267.

Tsyganenko, N. A., and D. H. Fairfield (2004), Global shape of the magnetotail current sheet as derived from Geotail and Polar data, J. Geophys. Res., 109, A03218, 11 pp., doi:10.1029/2003JA010062.

Vasyliūnas, V. M. (1983), Plasma distribution and flow, in Physics of the Jovian Magnetosphere, edited by A. J. Dessler, chap. 11, pp. 395-453, Cambridge Univ. Press, Cambridge, doi:10.1017/CBO9780511564574.013.

Vasyliūnas, V. M., L. A. Frank, K. L. Ackerson, and W. R. Paterson (1997), Geometry of the plasma sheet in the midnight-to-dawn sector of the jovian magnetosphere: Plasma observations with the Galileo spacecraft, Geophys. Res. Lett., 24(8), 869-872, doi:10.1029/97GL00757.

Waldrop, L. S., T. A. Fritz, M. G. Kivelson, K. Khurana, N. Krupp, and A. Lagg (2005), Jovian plasma sheet morphology: Particle and field observations by the Galileo spacecraft, Planet. Space Sci., 53, 681-692, doi:10.1016/j.pss.2004.11.003.

N. André, Institut de Recherche en Astrophysique et Planétologie, 9 av. du Colonel Roche, F-31028 Toulouse, France.

D. J. Andrews, S. W. H. Cowley, and G. Provan, Department of Physics and Astronomy, University of Leicester, University Road, Leicester LE1 7RH, UK

C. S. Arridge and A. J. Coates, Mullard Space Science Laboratory, University College London, Dorking RH5 6NT, UK. (csa@mssl.ucl.ac.uk)

M. K. Dougherty, Blackett Laboratory, Imperial College London, Prince Consort Road, South Kensington, London SW7 2AZ, UK.

C. M. Jackman, Department of Physics and Astronomy, University College London, Gower Place, London WC1E 6BT, UK.

K. K. Khurana and C. T. Russell, Institute for Geophysics and Planetary Physics, University of California, 603 Charles Young Dr. E., Los Angeles, CA 90024-1567, USA

E. C. Sittler, NASA Goddard Space Flight Center, MC 673, Greenbelt, MD 20771-0001, USA.

D. T. Young, Southwest Research Institute, 6220 Culbera Dr. San Antonio, TX 78238-0000, USA. 